\title{
40 AÑOS DE ACTA BOTANICA MALACITANA
}

\author{
A. Enrique SALVO TIERRA ${ }^{1 *}$, Antonio FLORES MOYA ${ }^{1}$, \\ Antonio GONZÁLEZ BUENO² y Baltasar CABEZUDO ${ }^{1}$ \\ ${ }^{1}$ Departamento de Biología Vegetal. Facultad de Ciencias. Universidad de Málaga \\ ${ }^{2}$ Departamento de Farmacia y Tecnología Farmacéutica. \\ Facultad de Farmacia. Universidad Complutense \\ *Autor para correspondencia: salvo@uma.es
}

Recibido el 18 de septiembre de 2014, aceptado para su publicación el 24 de octubre de 2014

RESUMEN. 40 años de Acta Botanica Malacitana. Se realiza una recensión de los 38 volúmenes de Acta Botanica Malacitana, así como de los dos números de Trabajos y Monografías del Departamento de Botánica. Así mismo se realiza un análisis bibliométrico y se construyen varios índices de autores, temáticas y novedades corológicas, taxonómicas y sintaxonómicas.

ABSTRACT. 40 years of Acta Botanica Malacitana. recession of the 38 volumes of Acta Botanica Malacitana and the two issues Trabajos y Monografias del Departamento de Botánica is performed. Also a bibliometric analysis is performed and several indexes of authors, subjects and chorological news, taxonomic and syntaxonomical are elaborate.

Acta Botanica Malacitana (ABM en adelante) tiene su origen en 1974 coincidiendo con el período de surgimiento de nuevas universidades "periféricas" en España. La numerosa población estudiantil en los campus universitarios clásicos, con la conflictividad que conllevaba, y la demanda de las principales capitales no universitarias a tener instituciones independientes, obligan al gobierno de la dictadura a un proceso descentralizador. De las 17 universidades, casi todas seculares, existentes en 1968, en una década se pasa al doble.

En los comienzos de la década de 1960 (fig. 1) las Facultades de Farmacia y Ciencias (con las recién creadas secciones de Biología) de las Universidades Complutense de Madrid, Barcelona, Valencia, Granada, Sevilla, Salamanca, Santiago de Compostela, Oviedo y La Laguna concentran, junto con los Jardines Botánicos de Madrid y de Barcelona, alrededor de doscientos profesionales dedicados a los estudios botánicos. Los catedráticos, investidos entonces de un poder omnímodo, crean sagas que compiten a partir de entonces por situar a delfines en las nuevas cátedras, agregadurías y adjuntías. La saga Rivas (Rivas Goday y Rivas Martínez) en la Complutense-Jardín 


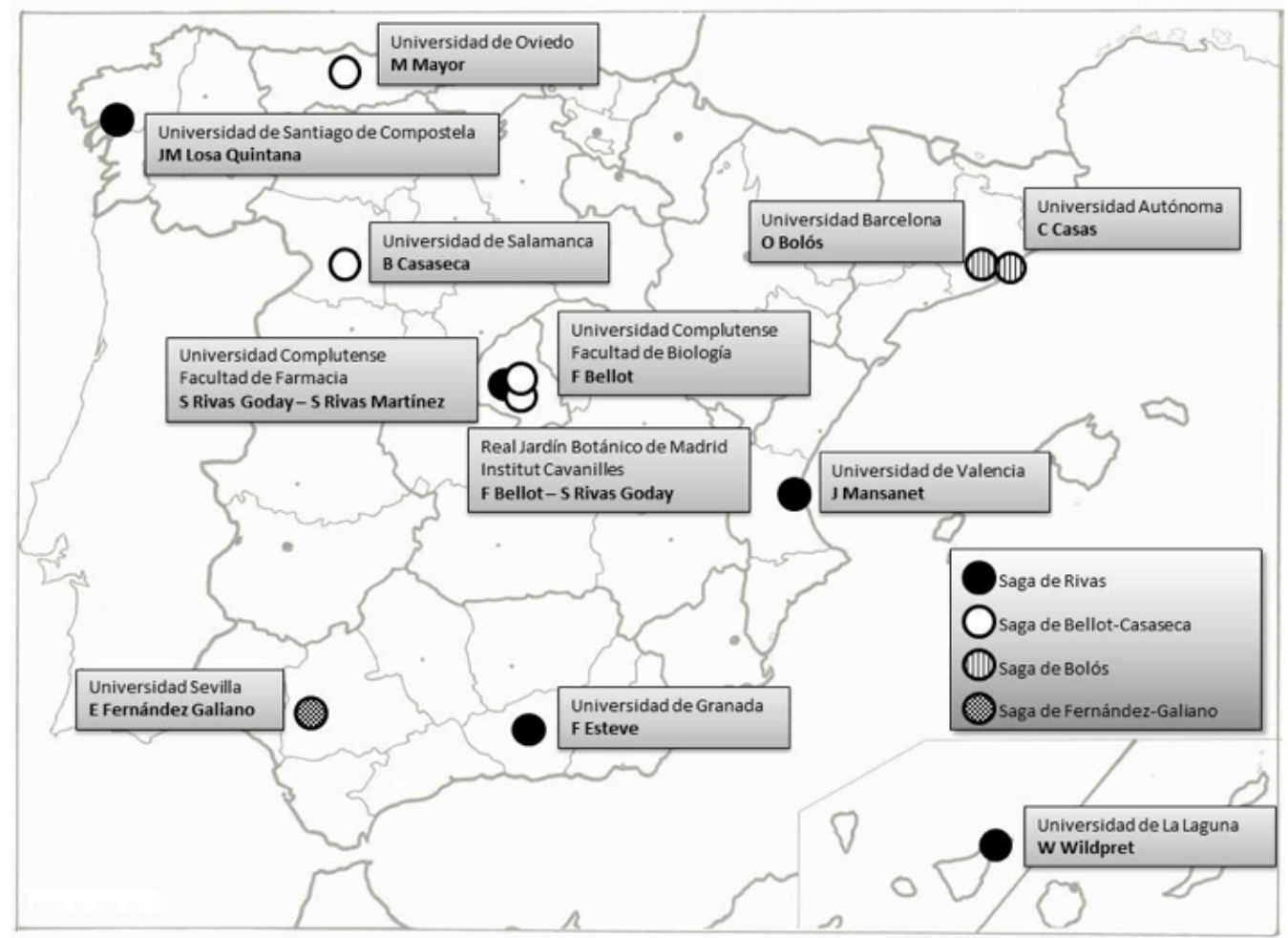

Figura 1. Núcleos de estudio de la botánica y sagas en España al inicio de la década de 1960.

Botánico irradia con fuerza entre los Profesores No Numerarios (PNNs) de los nuevos establecimientos académicos atraídos por una interesante metodología que crece con fuerza en nuestro país, la fitosociología. El tándem BellotCasaseca, desde Madrid-Salamanca, apuesta firmemente por el conocimiento florístico y así lo manifiestan en su docencia, investigación y, muy especialmente, en la dureza de los ejercicios "de visu" en las oposiciones para profesorado estable. En Barcelona, a la sombra del Institut Botànic, se desarrollan estudios fitosociológicos, como los emprendidos por Oriol de Bolòs, pero también cobran fuerza los criptogámicos, en especial los briológicos desarrollados por Casas. Fernández-Galiano, desde Sevilla, marca como prioridad los estudios florísticos, taxonómicos y de biología reproductiva en el mediodía peninsular, mientras Esteve-Chueca, desde Granada, hace lo propio pero desde la perspectiva fitosociológica. No obstante, la segregación del Departamento de la Facultad de Ciencias, con Varo a la cabeza, hace que surja un modelo transdisciplinar en donde ficología, micología, liquenología, briología, pteridología, florística y fitosociología son capaces de convivir y de colaborar. El Real Jardín Botánico vive en aquel momento cierta indefinición, con un cierre por obras que parecía concluyente, lo que hace reaccionar a sus investigadores para que, en la década de los 80 , ocurra un trascendente renacimiento.

Los Anales del Jardín Botánico de Madrid alcanzan la treintena de volúmenes siendo, 


\begin{tabular}{|c|c|c|c|}
\hline & Inicio & Indice h5 & Mediana h5 \\
\hline Anales del Jardín Botánico de Madrid & 1941 & 7 & 9 \\
\hline Collectanea Botanica & 1946 & & \\
\hline Lagascalia & 1971 & & \\
\hline Acta Botanica Malacitana & 1975 & 6 & 8 \\
\hline Botánica Macaronésica & 1976 & & \\
\hline Acta BotanicaBarcinonensia & 1978 & & \\
\hline Boletín de Ciencias de la Naturaleza & 1979 & & \\
\hline Lazaroa & 1979 & 4 & 8 \\
\hline Fontqueria & 1982 & & \\
\hline Studiabotanica & 1982 & 5 & 5 \\
\hline Blancoana & 1983 & & \\
\hline Anales de Biología. Sección Biología Vegetal & 1987 & & \\
\hline Itinera Geobotanica (Asociación Española de Fitosociología) & 1987 & & \\
\hline Botanica Complutensis & 1989 & 4 & 4 \\
\hline Revista Iberoamericana de Micología & 1990 & 15 & 23 \\
\hline Archivos de Flora Ibérica & 1991 & & \\
\hline Boletín de la Sociedad Española de Briología & 1993 & & \\
\hline Monografias del Jardín Botánico de Córdoba & 1994 & & \\
\hline Boletín de la Asociación de Herbarios Ibero-Macaronésicos & 1995 & & \\
\hline Flora Montiberica & 1995 & 3 & 4 \\
\hline Guineana & 1995 & & \\
\hline Polen & 1995 & & \\
\hline Revista Catalana de Micologia & 1995 & & \\
\hline Boletin BIGA & 2006 & & \\
\hline Bouteloua & 2006 & & \\
\hline El/0 botânico: Revista de la AIMJB & 2007 & & \\
\hline Folia Botanica Extremadurensis & 2007 & & \\
\hline
\end{tabular}

Tabla 1. Revistas de contenido exclusivamente botánico editadas en España. Indice h5 y Mediana h5 basado en Ayllón, Juan Manuel; Ruiz Pérez, Rafael, ; Delgado López-Cózar, Emilio (2013). Hi: Índice H de las revistas científicas españolas según Google ScholarMetrics (2008-2012). EC3 Reports, 7. Granada, 18 de noviembre de 2013. Corresponding.

junto a Collectanea Botanica editada por el Instituto Botánico de Barcelona, las únicas revistas españolas dedicadas a la botánica. A partir de los 70 aparecen hasta una treintena de nuevas publicaciones (tab. 1), algunas de ellas efímeras, como fue el caso de los Trabajos y monografías de Botánica de la Universidad de Granada (1976-1984). El mundo de las revistas internacionales aún quedaba muy lejano para los investigadores españoles.

Así ABM (tab. 2) se funda en 1974, año de la creación por Decreto de la Facultad de Ciencias de la Universidad de Málaga (UMA), viendo su primer número impreso en 1976 ("Se termina de imprimir en 1976 con ISBN 84-500-7342-1 y Dep. Legal MA 46-1976. Imprime Gráficas Urania") pero con año de publicación de 1975. En aquel momento, el Departamento de Botánica se reducía a una plantilla de cuatro profesores: Alfredo Asensi Marfil, Blanca Díez Garretas, Ángel M. Hernández Cardona y J. Carlos Escudero. El volumen, impreso en tipografía y sin Comité Editorial, aparece como una publicación "Editada por el Excmo Ayuntamiento de Málaga con la colaboración de la Facultad de Ciencias de la Universidad de Málaga" y así, en su portada en verde 


\begin{tabular}{|l|l|c|}
\hline $\begin{array}{l}\text { Nombre correcto } \\
\text { Nombre incorrecto }\end{array}$ & $\begin{array}{c}\text { Acta Botanica Malacitana } \\
\text { Acta Botánica Malacitana }\end{array}$ & $\begin{array}{c}\text { La tilde en Botanica es incorrecta, ya que } \\
\text { el título es en latín y en esa lengua la tilde } \\
\text { no existe. }\end{array}$ \\
\hline Abreviatura correcta & Acta Bot. Malacitana & $\begin{array}{c}\text { Las abreviaturas no recomendadas son las } \\
\text { que inducen a error o confusión }\end{array}$ \\
\hline Abreviaturas no recomendadas & $\begin{array}{l}\text { Acta Bot. Mal. } \\
\text { Acta Bot. Malac. } \\
\text { Acta Bot. Malacit. }\end{array}$ & \\
\hline ISSN edición impresa & $0210-9506$ & \\
ISSN edición electrónica & $2340-5074$ & \\
\hline
\end{tabular}

Tabla 2. Nombres y abreviaturas correctas e incorrectas para ABM e ISSNs.

amarillento (PANTONE 375), se inscribe centralmente el escudo del Ayuntamiento de Málaga. El índice de paginación aparece en contraportada recogiendo un total de 8 artículos.

ABM se presentaba a la comunidad científica de esta forma:

"Se inicia con este volumen la publicación de ACTA BOTANICA MALACITANA que acogerá básicamente trabajos de Flora y Vegetación.

La revista tendrá una periodicidad anual y quedan invitados a colaborar todos los autores españoles y extranjeros que lo deseen.

Málaga, noviembre de 1975"

La tirada inicial, así como para varios números posteriores, fue de 300 ejemplares, además de 50 en forma de separatas para los autores.

El vol. II (1976) recogía 7 artículos y se incluía Notas para los autores. la dirección de referencia era el Colegio Universitario de Málaga (dependiente de la UGR y no de la UMA) ya que, aunque la UMA fue creada en 1972 con los estudios de Económicas y Medicina, las titulaciones de Ciencias (creada en 1974) dependían aun de la universidad granadina.

El vol. III (1977) presenta tres novedades significativas: se adscribe ABM como publicación del Instituto Botánico del Excmo. Ayuntamiento de Málaga, proyecto inconcluso que pretendía aunar universidad y un futurible Jardín Botánico Municipal; aparece un Comité de Redacción integrado sólo por dos directores (A. Asensi y B. Díez Garretas) y se tasan las suscripciones en "400 pesetas para España y 500 ptas para el extranjero".

En 1978 la edición del vol. IV pasa a la Imprenta de la UMA y así, en la parte posterior de la cubierta, figura el Secretariado de Publicaciones con un emblema de la paloma de Picasso en vuelo rodeada por la leyenda “Universitas Malacitana”, y se cambia el color corporativo a la referencia PANTONE 369.

El vol. V (1979) recoge la primera entrega de tres de las actas del III Simposio de Criptogamia celebrado en Málaga en 1978.En portada cambia el emblema del Ayuntamiento por el de la Diputación de Málaga, comprometida con la financiación de la publicación de las actas. En la trasera de cubierta se explicita que es editada por el Departamento de Botánica de la UMA. Se distingue un Comité de Dirección integrado por los dos directores (A. Asensi y B. Díez Garretas) al que se añade un Secretario de Redacción (J. Guerra), además de un Comité Asesor compuesto por 13 especialistas, entre otros los miembros del Departamento: T.E. Díaz González y A. E. Salvo y, más tarde, F. 


\section{Directores}

\begin{tabular}{ccc}
\hline $\begin{array}{c}\text { A Asensi (1977-1979) } \\
\text { B Cabezudo (1982-2013) (Editor en Jefe en la estructura entre 2004-2012) } \\
\text { Secretarios }\end{array}$ & B.Díez Garretas (1977-1979) \\
\hline J Guerra (1979-1986) & AE Salvo (1987-1989) \\
JM Nieto (1989-1997) & F Conde (1998-2003) \\
\hline MM Trigo (2004-2012) & Vicesecretarios \\
\hline F Conde (1987-1997) & JM Nieto (1998) \\
AE Salvo (1991, 1995-1997) & MM Trigo (1998-2003) \\
Comité editor (Editores asociados entre 2004-2012)
\end{tabular}

AE Salvo (1991, 1998-2001, 2012-2013)

A Flores (1993-2013)

AV Pérez Latorre (1995-2013)

F Conde (2004-2005)
MM Trigo (1991-2007, 2013)

T Navarro (1993-2013)

JM Nieto (1998-2003)

M Altamirano (2013)

Editora electrónica

M Recio (2007-2013)

\section{Comité Asesor Permanente}

FD Calonge (1982-2012)

C Casas (1982-2012)

M Costa (1982-2012)

A Crespo (1982-2012)

TE Díaz (1982-2013)

J Izco (1982-2013)

X Llimona (1982-2012)

C Sáenz (1982-2011)

J Seoane (1982-2012)

B Valdés (1982-2013)

AE Salvo (1982-1986)

S Rivas Martínez (1982-2013)

E Nardi (1983-2013)

S Talavera (1983-2013)

J Varo (1983-2012)
G López (1986-2013)

JP Herbrard (1986-2013)

C Favarger (1986-2013)

JL Pérez Cirera (1986-2012)

J Guerra (1987-2013)

JA Devesa (1994-2013)

P Sánchez (1994-2013)

E Domínguez (1995-2013)

G Blanca (1996-2013)

C Herrera (1999-2013)

M Fennane (2000-2013)

E Dana (2013)

A Galán de Mera (2013)

F Mota (2013) 


\begin{tabular}{|c|c|c|}
\hline Secciones fijas & Editores & Volumen Inicio (año) \\
\hline Notas pteridológicas & AE Salvo / B Cabezudo & XI (1986) \\
\hline $\begin{array}{l}\text { Fragmentos taxonómicos, corológicos, } \\
\text { nomenclaturales y fitocenológicos }\end{array}$ & $\begin{array}{l}\text { JM Nieto / T Navarro /AV Pérez Latorre } \\
\text { / A Flores }\end{array}$ & XII (1987) \\
\hline $\begin{array}{l}\text { Botanicae Historiae Schedulae Sparsae - Notas } \\
\text { históricas }\end{array}$ & AI González Bueno & XIII (1988) \\
\hline Fragmenta Palynologica Baetica & G Blanca / MM Trigo & XIII (1988) \\
\hline $\begin{array}{l}\text { Contribuciones a la flora vascular de Andalucía } \\
\text { (España) y del Rif (Marruecos) }\end{array}$ & C Morales / B Cabezudo / F Mota & XIV $(1989)^{*}$ \\
\hline Novedades taxonómicas y sintaxonómicas & T Navarro / AV Pérez Latorre & XXIII $(1998) * *$ \\
\hline $\begin{array}{l}\text { Contribuciones a la flora vascular de Andalucía } \\
\text { (España) }\end{array}$ & C Morales / B Cabezudo & XXV (2000) \\
\hline $\begin{array}{l}\text { Contribuciones a la flora vascular del Rif } \\
\text { (Marruecos) }\end{array}$ & M Fennane / J El Oualidi & XXV (2000) \\
\hline $\begin{array}{l}\text { Notulae taxinomicae, chorologicae, } \\
\text { nomenclaturales, bibliographicae aut } \\
\text { philologicae opus "flora iberica intendentes }\end{array}$ & S Castroviejo / JA Devesa & XXX (2005) \\
\hline
\end{tabular}

* Hasta el vol XXIV (1999) en que se subdivide en dos secciones

** Hasta el vol XXXIV (2009) en que se incluye en Fragmentos taxonómicos

Tabla 3. Secciones de ABM.

Conde, con lo que los seis componentes del Departamento en aquel momento formaron parte del proyecto editorial.

En 1980 se edita el vol. VI con una segunda entrega de ponencias del III Simposio Criptogamia. En este número no aparece Comité de Redacción.

Aún quedaba un tercer bloque de ponencias y el compromiso de la Diputación de Málaga de correr con los cargos de la edición de todas las Actas del Simposio se incumple. Es un momento difícil en España; a la agitada transición política se le añade una profunda crisis económica, muy especialmente institucional, lo que complica el futuro de los proyectos editoriales. Esta paralización obliga al Departamento a sacar una nueva publicación "lowcost": Trabajos y Monografías del Departamento de Botánica de la Universidad de Málaga (TyM), con la que dar respuesta al compromiso adquirido con los autores que daban por recepcionados y aceptados sus artículos. Los dos números de Ty $M$ fueron editados artesanalmente mediante una máquina de escribir electrónica Olivetti ET 2400 y su posterior impresión en offset. Los volúmenes 1 y 2 aparecieron, sucesivamente, en 1980 y 1981. En este segundo se rinde homenaje a Salvador Rivas Goday y Modesto Laza Palacios, ambos fallecidos en 1981.

La convocatoria de plazas de profesores numerarios (adjuntos y agregados) provoca una remodelación y una movilidad de profesores 
sin precedente en la universidad española. Así, en la Universidad de Málaga, recala Baltasar Cabezudo, quien sería su primer Catedrático de Botánica. Cabezudo, originario de la saga sevillana de Fernández-Galiano, se impone dos metas: consolidar el Herbario MGC como uno de los referentes de la flora española y conseguir que $A B M$ se constituya en una revista con periodicidad anual y competitiva desde el punto de vista de la calidad de su contenido. Desde este eje vertebrador permite la innovación y la flexibilización, desde el diseño a distintas fórmulas de Comité Editorial, pasando por la amplitud de temáticas y territorios.

El 17 de Septiembre de 1981 el Boletín Oficial de Estado hace oficial la creación del Departamento de Botánica de la UMA.

A partir de 1982 ABM recupera su edición anual normalizada. El vol. VII recoge el último bloque de artículos correspondiente al Simposio de Criptogamia de 1978. La edición fue sufragada conjuntamente con el Colegio Oficial de Farmacéuticos de Málaga, al dedicarse el volumen a la memoria de los farmacéuticos Modesto Laza Palacios "insigne botánico malagueño" y José María Laza Rojas, "primer profesor que fue del Departamento de Botánica de la Facultad de Ciencias de la Universidad de Málaga". Varios avances significativos se producirán con este número: el logo de la UMA aparece en portada, quedando de manifiesto que se trataba de una publicación universitaria; se consolida un Comité de Redacción compuesto por un Director (B. Cabezudo), un Secretario (J. Guerra) y un Comité Asesor compuesto por 12 profesores de distintas Instituciones; por último, aparecen unas normas de publicación detalladas.

En el vol. VIII (1983) se recogen las ponencias de la Reunión Internacional de Pteridología celebrada en Algeciras en Octubre de 1980, otorgando un gran prestigio a la revista por la excelencia de su contenido. La metodología seguida para la edición de este número, así como hasta el vol. XIII, fue la misma que para $T y M$, dado el ahorro en costes que suponía en una coyuntura tan difícil.

En el vol. XI (1986) se abren por primera vez secciones fijas (tab. 3) con Notas Pteridológicas.

El vol. XII (1987) está dedicado a Edmond Boissier, con motivo del 150 aniversario de su viaje por el Sur de España, así como a los dos botánicos malagueños que jugaron un papel trascendental en sus investigaciones: Pablo Prolongo y Félix Haenseler. Se lleva a cabo una importante remodelación: desde el cambio de portada, que incluye por primera vez un elemento florístico, hasta la reestructuración del Comité editor y del Comité Asesor, adquiriendo un carácter internacional, además de abrir la nueva sección Fragmentos Taxonómicos, Corológicos, Nomenclaturales y Fitocenológicos. Pero lo más significativo es que ABM aparece por primera vez adscrita al Departamento de Biología Vegetal de la UMA, ya que en ese año, y como consecuencia de la aplicación de la Ley de Reforma Universitaria, las áreas de conocimiento de Botánica y Fisiología Vegetal se fusionan en una sola: Biología Vegetal, de la que toma su nombre el Departamento. Aquella fusión no contentaba a ninguna de las dos partes, ya que eran disciplinas a priori diametralmente opuestas, la fisiológica más experimental mientras que la botánica, aún muy asentada en una florística de campo, era de observación y de carácter regional. Especialmente para el medio millar de profesionales de la botánica española aquello suponía el golpe definitivo de la botánica clásica en favor de los métodos bioquímicos y fisiológicos. Las consecuencias no fueron tan traumáticas; la convivencia fue amoldándose y los ámbitos de trabajo fueron respetados. Curiosamente 25 años después, cuando la aproximación de ambas disciplinas es cada vez mayor, se ha producido una desunión distantemente deseada.

La portada de 1987 sirvió de base para que en el vol. XIII se diera con el motivo principal 


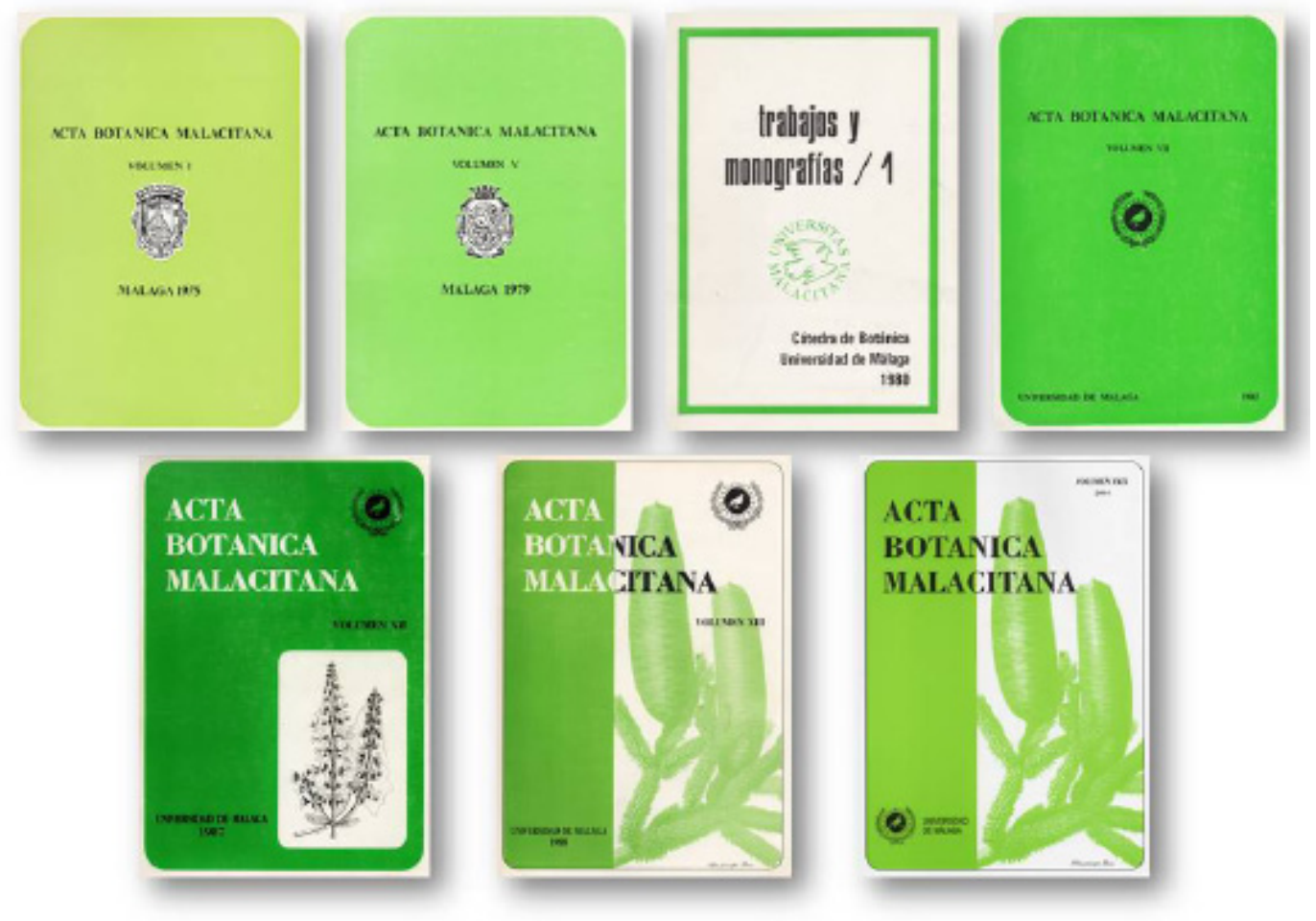

Figura 2. Evolución de la portada de ABM y TyM.

para una imagen corporativa de la revista: el pinsapo (fig. 2). Dos nuevas secciones fijas se incorporaron: Botanicae Historiae Schedulae Sparsae y Fragmenta Palynologica Baetica.

En 1989 (vol. XIV), coincidiendo con la adquisición de material informático, la revista pasa a editarse digitalmente, teniendo su arte final directamente en imprenta. Se incorpora la sección Contribuciones a la Flora vascular de Andalucía y el Rif.

El vol. XV (1990), el más voluminoso (373 pags.), y el XVI (1991), desagregado en dos fascículos, incorporan en su mayor parte las comunicaciones presentadas en el Simposio de Criptogamia celebrado en Melilla y organizado por el Departamento, y suponen un importantísimo salto en calidad de la revista. ABM se consolida en ese momento como la revista española de mayor proyección criptogámica. Asimismo, se recogen las contribuciones de las VIII Jornadas de Fitosociología celebradas en Málaga en 1988.

El vol. XXI de 1996 se dedica a la memoria de Josep Cuatrecasas y Tadeus Reichstein.

Por entonces aparece un factor que modulará la producción científica en España a través de la aprobación de complementos salariales de investigación (sexenios) basada en artículos publicados en revistas con factor de impacto. Este aliciente para que la ciencia española se universalice provoca, simultáneamente, un debilitamiento de las revistas mayoritariamente en lengua española en favor de las revistas anglosajonas con índices de impacto mucho más relevantes. 
En la Botánica Española tan sólo Anales del Jardín Botánico y Revista Iberoamericana de Micología tienen reconocido este factor. En este mismo sentido se produjo, a la vez, un vuelco masivo de recursos humanos y económicos hacia las nuevas tecnologías de la Información y la Comunicación, en detrimento de áreas del conocimiento como la Botánica, de escasa rentabilidad inmediata. Desde entonces y hasta ahora, los profesionales dedicados a registrar la diversidad vegetal han disminuido en España, ya que no solamente no se han creado nuevas plazas sino que tampoco se amortizan la de los que egresan. Este fenómeno, que también se está dando en otros países cómo el Reino Unido o Estados Unidos, caló profundamente desde un principio en la comunidad botánica española que ha ido abandonando los procedimientos de catalogación para pasar a buscar soluciones a interrogantes mediante metodologías cada vez más sofisticadas. El cambio en la producción botánica es más que patente a través de las páginas de $\mathrm{ABM}$.

El vol. XVIII incluye una página final sobre la Base de Datos ICYT del Consejo Superior de Investigaciones Científicas, dando con ello a entender su incorporación al repositorio español más importante. Asimismo, en el vol. XXIII (1998), en los créditos de contraportada, se lee: "Accredited with the International Association for Plant Taxonomy for the purpose of registration of all new plant names". La edición de este volumen tiene una singularidad al pasar del formato de una sola columna por página, a dos columnas y también se indica, por primera vez, una dirección electrónica para acceder libremente a la versión accesible de la revista en la red.

El vol. XXVII (2002) está dedicado a la memoria de Elisabeth (Betty) Eleanor Molesworth Allen y el XXX (2005) a la de Francisco Conde, compañero del Departamento y miembro activo en la edición de la revista durante todos estos años. También se dedican obituarios en volúmenes posteriores dedicados a Emilio Fernández Galiano y Suzette Puech (2006), Bárbara Aru (2008), Santiago Castroviejo Bolívar, César Gómez Campo y Oswaldo Socorro (2009) y Rafael Suau (2010). Todos ellos colaboraron con mayor o menor intensidad, pero siempre con mucha ilusión con ABM.

Desde el vol. XXXII (2007) la revista incluye entre las Instrucciones para los autores una nota en la que se explicita: "Los trabajos, una vez aceptados, se editarán en versión electrónica en la página http:// www.uma.es/Estudios/Departamentos/ BiolVeg/03Rev/00HRev/01Rev.html".

En los volúmenes XXXIV (2009) y XXXV (2010), en los créditos, se incluye una llamada significativa: Indexada en: Agris, Biosis, Dialnet, e-Revistas, ISI Master JournalList, Latindex, Previews (R) and Scopus.

En el vol. XXXVI (2011) se interrumpen las suscripciones a la edición impresa de la revista, que solo se puede obtener, a partir de entonces, por peticiones institucionales e intercambios con otras publicaciones, o mediante el intercambio con exsiccatas de herbario.

El vol XXXVIII (2013) introduce cambios de importancia como la incorporación del ISSN de la versión electrónica (2340-5074), la modificación de las cabecillas de los trabajos motivada por las recomendaciones de Latindex $\mathrm{y}$, en el apartado de Instrucciones a los autores, la incorporación de un último párrafo sobre la evaluación de los manuscritos recepcionados, en primera instancia, por el Comité Editorial.

Ayllón et al. (2013) sitúan a ABM cómo la séptima en el ranking de revistas españolas de Ciencias Biológicas, y la tercera de las dedicadas a Botánica, tras la Revista Iberoamericana de Micología y los Anales del Jardín Botánico de Madrid.

\section{ESTUDIO BIBLIOMÉTRICO}

ABM (incluyendo aquí TyM) ha publicado 


\begin{tabular}{|c|c|c|c|c|c|c|c|c|}
\hline Artículos & $\begin{array}{c}\text { Notas } \\
\text { Pteridológicas }\end{array}$ & $\begin{array}{c}\text { Notas } \\
\text { Históricas }\end{array}$ & $\begin{array}{c}\text { Fragmentos } \\
\text { Taxonómicos } \\
\text { Corológicos }\end{array}$ & $\begin{array}{c}\text { Fragmenta } \\
\text { Palynologica }\end{array}$ & $\begin{array}{c}\text { Contribuciones } \\
\text { Flora } \\
\text { Andalucía }\end{array}$ & $\begin{array}{c}\text { Contribuciones } \\
\text { Flora } \\
\text { Marruecos }\end{array}$ & $\begin{array}{c}\text { Reseñas } \\
\text { Bibliográficas }\end{array}$ & $\begin{array}{c}\text { Notas } \\
\text { Flora } \\
\text { Ibérica }\end{array}$ \\
\hline $\mathbf{5 3 2}$ & $\mathbf{5 7}$ & $\mathbf{5 0}$ & $\mathbf{2 4 8}$ & $\mathbf{1 1}$ & $\mathbf{1 5 7}$ & $\mathbf{2 0}$ & $\mathbf{7}$ & $\mathbf{2 5}$ \\
\hline
\end{tabular}

Tabla 4. Número de títulos por sección en ABM.

en estos 40 años 1.111 títulos (tab. 4) recogidos en 10.271 páginas, de los que 532 corresponden a artículos y el resto a notas de las distintas secciones.

Por temática (tab. 5) los trabajos florísticos suponen algo más de la cuarta parte de las aportaciones publicadas, seguidos de los taxonómicos de fanerógamas, los fitosociológicos, los pteridológicos y los ficológicos.

Así se han publicado en ABM hasta el momento 363 novedades corológicas (tab. 6), 182 taxonómicas (Anexo II) y 468 sintaxonómicas (Anexo III).

\begin{tabular}{|c|c|c|}
\cline { 2 - 3 } \multicolumn{1}{c|}{} & $\mathrm{N}^{\mathrm{o}}$ Art & $\%$ \\
\hline Floristica & 290 & 26,10 \\
\hline Taxonomía & 157 & 14,13 \\
\hline Fitosociología & 120 & 10,80 \\
\hline Pteridología & 108 & 9,72 \\
\hline Ficología & 106 & 9,54 \\
\hline Palinología & 69 & 6,21 \\
\hline Historia & 66 & 5,94 \\
\hline Micología & 47 & 4,23 \\
\hline Briología & 46 & 4,14 \\
\hline Liquenología & 33 & 2,97 \\
\hline Conservación & 15 & 1,35 \\
\hline Fenomorfología & 19 & 1,71 \\
\hline Ecología & 11 & 0,99 \\
\hline Anatomía & 6 & 0,54 \\
\hline Fitoquimica & 5 & 0,45 \\
\hline Otros & 13 & 1,17 \\
\hline
\end{tabular}

Tabla 5. Número de artículos por temática en ABM.
La Península Ibérica es el ámbito territorial (tab. 7) más usado (21,0\%), seguido por Andalucía (12,7\%), España (9,5\%), Málaga $(6,0 \%)$, Marruecos $(5,5 \%)$ y Galicia $(3,4 \%)$.

La lengua española es la más extendida entre las aportaciones, aunque un 12,6\% han utilizado otros idiomas. El número de artículos publicados, según idiomas usados, son: inglés: 65, francés: 41, portugués: 27 , italiano: $6 \mathrm{y}$ alemán: 1 .

Mil setenta y seis autores han contribuido con aportaciones (Anexo I - Índice de autores). Prácticamente, en dicho elenco aparecen todos los autores botánicos españoles de estos últimos 40 años. De ellos, 93 han publicado más de 5 contribuciones, de los que más del $80 \%$ no son miembros del Departamento de Biología

\begin{tabular}{|l|c|}
\hline \multicolumn{2}{|c|}{ NOVEDADES COROLÓGICAS } \\
\hline España & 99 \\
\hline Andalucía & 78 \\
\hline Marruecos & 74 \\
\hline Península Ibérica & 53 \\
\hline Europa & 18 \\
\hline Canarias & 12 \\
\hline Portugal & 10 \\
\hline Mediterráneo & 5 \\
\hline Brasil & 4 \\
\hline Macaronesia & 4 \\
\hline Baleares & 3 \\
\hline Africa & 1 \\
\hline Neotrópico & 1 \\
\hline
\end{tabular}

Tabla 6. Novedades corológicas, por áreas, publicadas en ABM. 


\begin{tabular}{ccc}
\hline & $\mathrm{N}^{\mathrm{o}}$ de autores & $\%$ \\
\hline Miembros No Dpto Top+5 & 76 & 81 \\
Miembros del Dpto Top+5 & 18 & 19 \\
\hline
\end{tabular}

Tabla 7. Proporción de autores con más de 5 publicaciones que no han sido miembros del departamento editor, y los que si.

Vegetal de la UMA, editor de la revista (tab. 8).

La media del número de artículos publicados por año se sitúa en 28,4, alcanzando su máximo en 1991 con 51 artículos (fig. 3).

El número medio de autores por artículo es de 2,4 con una desviación típica de 0,5. Es llamativo que a lo largo de estos 40 años se manifiesta un incremento en el número de autores por artículo, duplicándose la media desde $1975(1,63)$ hasta los diez últimos volúmenes en donde se ha superado la media de 3 en 4 volúmenes (fig. 4).

La distribución del número de autores por artículo (fig. 5) alcanza hasta los 15 y tiene una distribución que se ajusta a una curva logarítmica, con una moda de 375 artículos con dos firmantes.

AGRADECIMIENTOS. Los autores, en nombre del equipo editorial y en el suyo propio, quieren testimoniar su agradecimiento a $\mathrm{M}^{\mathrm{a}}$ Dolores Tirado, Eva Linero, Rafael Gutiérrez, Luisa Gil y Daniel Moreno por su inestimable trabajo en la edición, administración y distribución de ABM.

Asimismo ha sido fundamental a lo largo de estos años el apoyo institucional a través del SPICUM (Servicio de Publicaciones e Intercambios de la Universidad de Málaga).

Finalmente, ABM quiere agradecer a los autores y asesores la confianza depositada en nuestra revista. Sin la alta cualificación de sus trabajos, sin su interés altruista y sin su permanente disponibilidad estos cuarenta años no hubiesen sido posible.

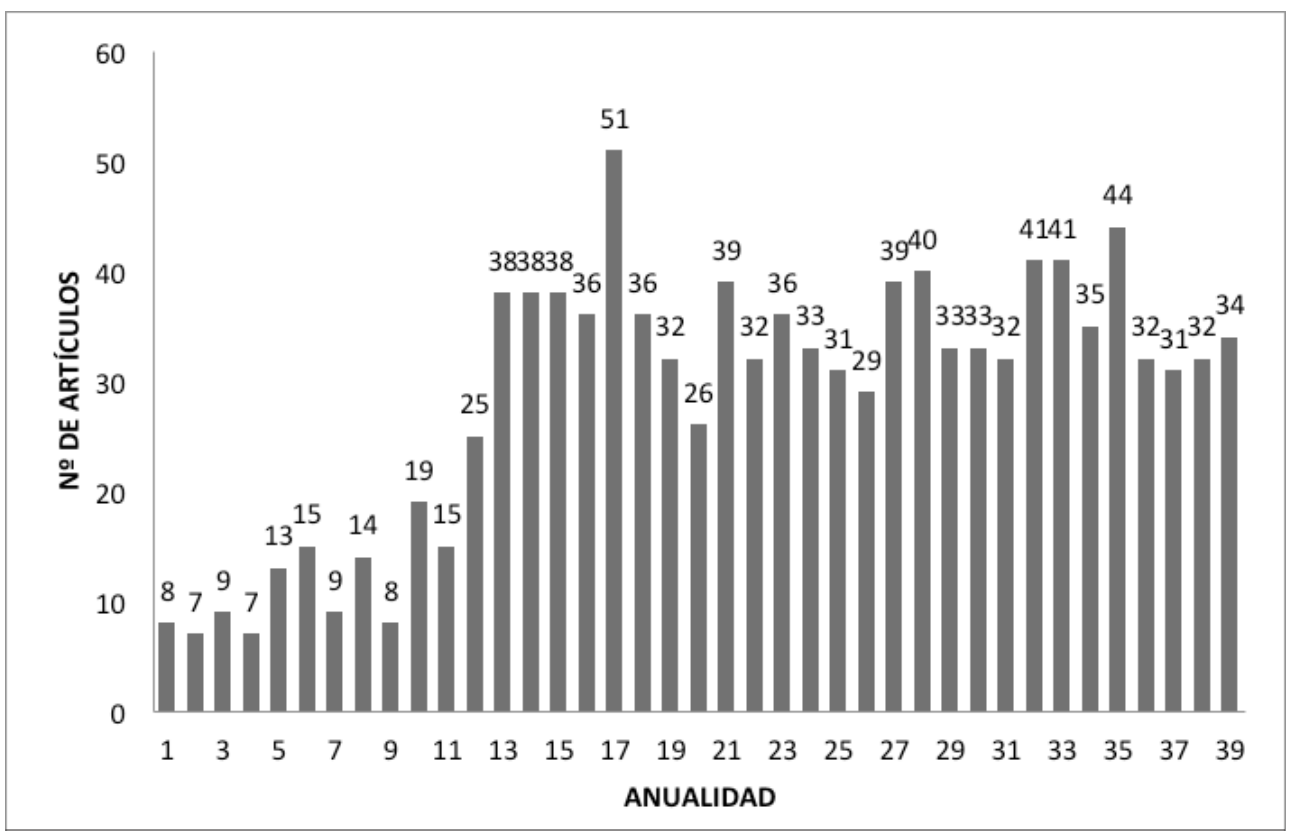

Figura 3. Evolución del número de artículos por año. 


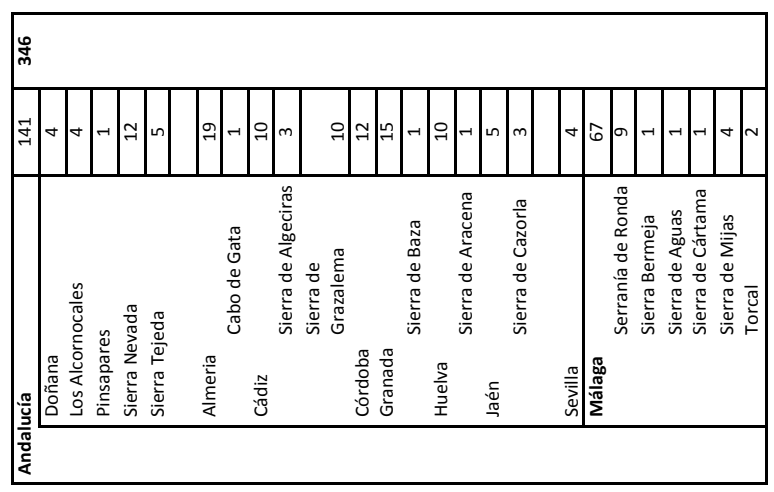

ஜ

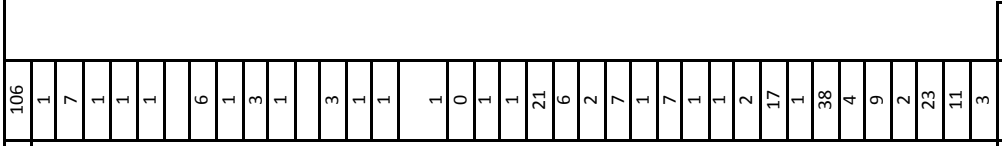

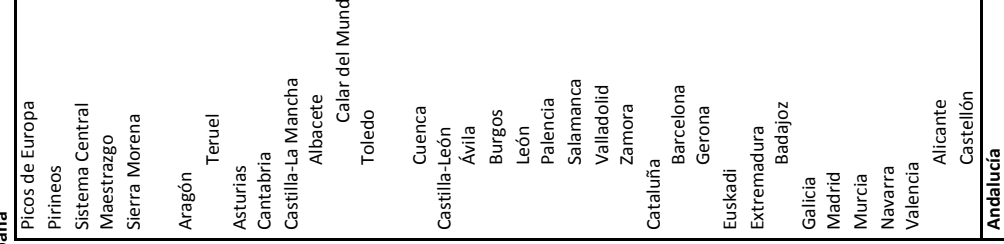

啇

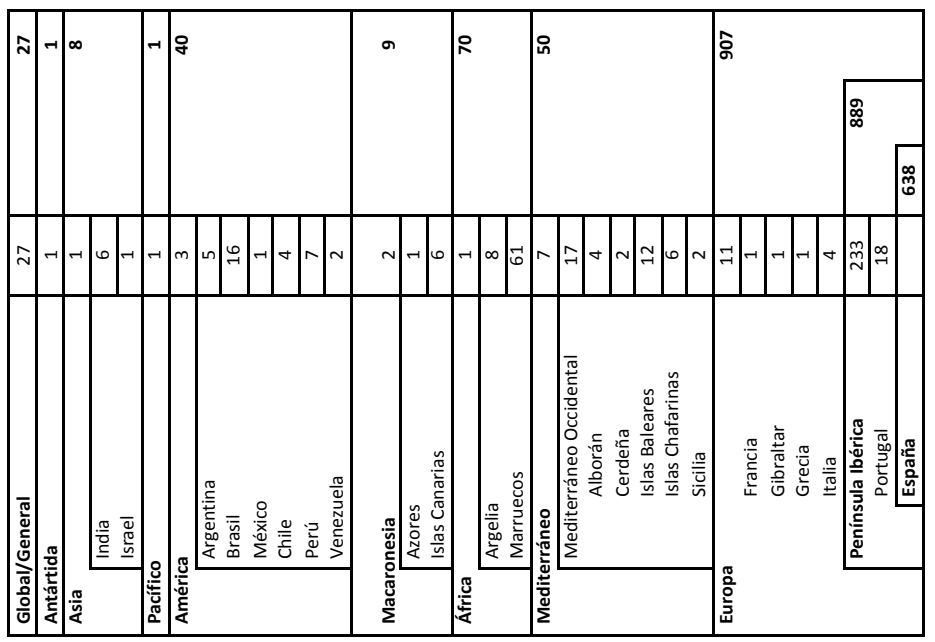

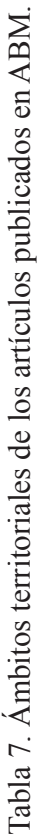




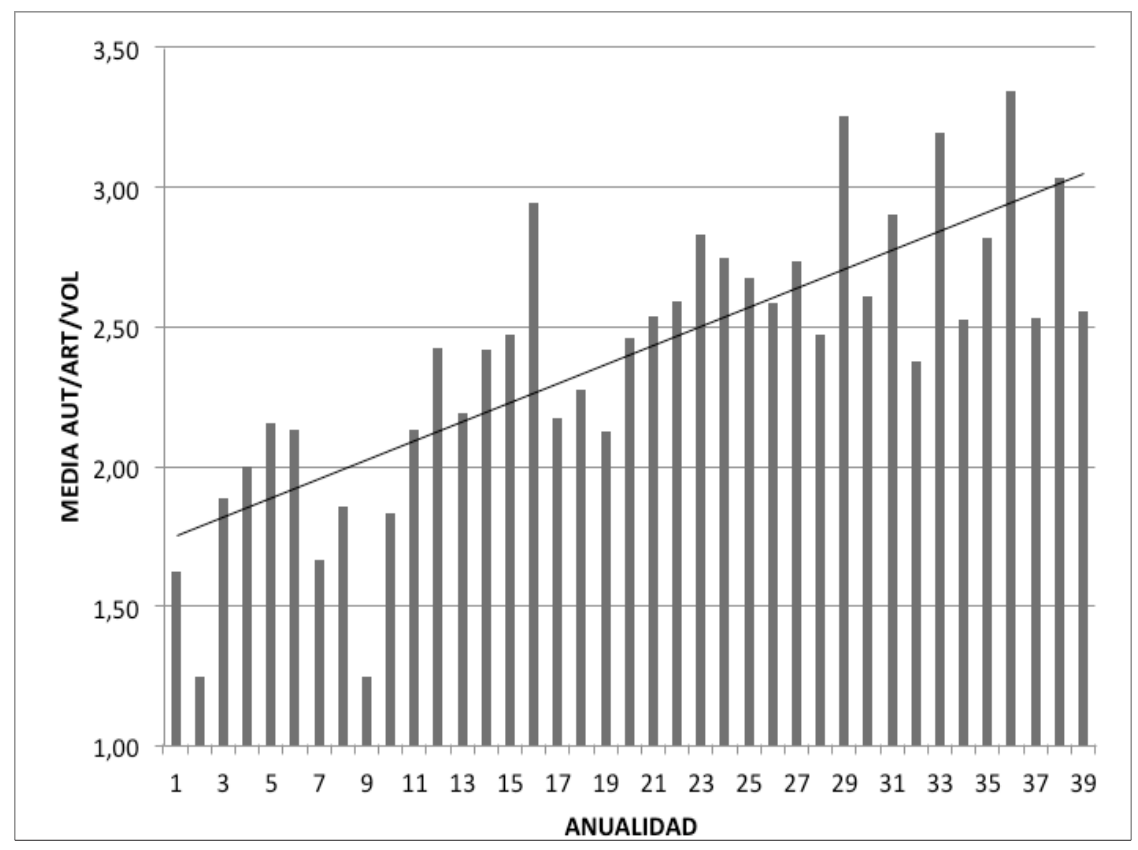

Figura 4.- Media del número de autores por artículo y volumen y línea de tendencia calculada por ajuste lineal por mínimos cuadrados.

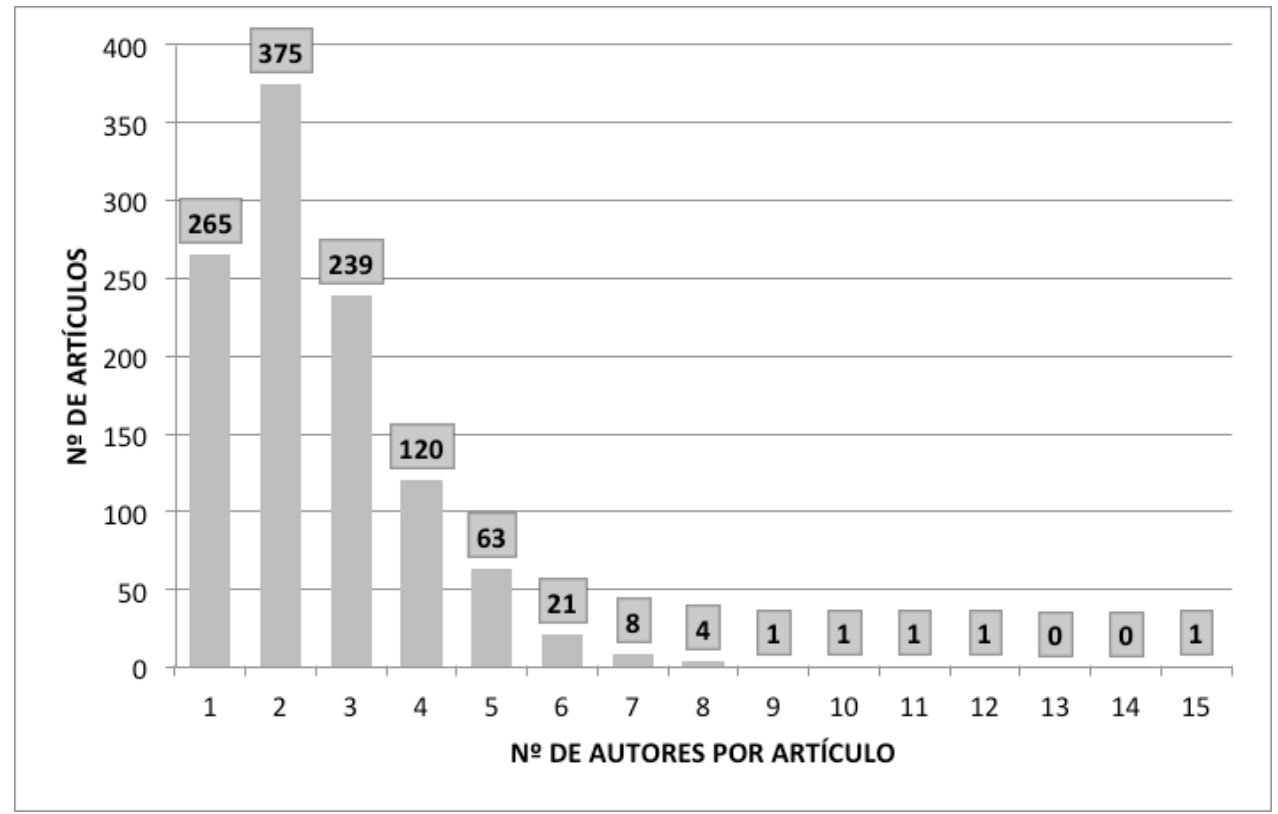

Figura 5. - Distribución del número de autores firmantes de artículos. 


\section{ANEXO I \\ INDICE DE AUTORES SEGUIDO DEL NÚMERO DE LOS VOLÚMENES EN LOS QUE APARECEN SUS PUBLICACIONES}

Aafi A 30

Aboal M 12141516193536

Abourouh M 35

Acedo C 2733

Acherkouk M 36

Achhal El Kadmiri A 2930

Acón Remacha M 18

Acosta A 35

Aedo C 133135

Aguiar C 29

Aguilera AM 27

Aira Rodríguez MJ 151718222427

Ajbilou R 32

Alaoui Haroni S 34

Alba Villegas S 32

Albarreal Núñez J 29

Alburquerque A 35

Alcaraz Ariza F 1213171819202129

Alcaraz M 7

Aleffi M 23

Alfiriqui M 34

Algarra Ávila JA 27

Alomar G 1112

Alonso FL 14192122

Alonso Herrero E 24

Alonso MA 22272935

Alonso Rendodo R 23242729333435

Alonso S 35

Alsina M 13

Altamirano M 19202122243133343538

Álvarez Calviño R 6

Álvarez Cobelas M 111213

Álvarez Fernández I 20

Álvarez J 27

Álvarez JM 18

Amado A 29

Amich F 2933

Amigo J 15

Amor Morales A 1534

Amouri AA 37

Amuchástegui 28

Andrés C 2628

Andrés Rodríguez J 3616

Andrés Ros JV 2628

Androusse L 32

Angulo Romero J 1718

Aparicio A 11121820222728

Aparicio J 27

Aparicio Rojo M 33

Arán Redo VJ 36

Aránega Jiménez R 30

Arauzo M 12

Arenas Posada JA 17

Arista M 171819202832
Arjona MMF 36

Armada J 30

Arnelas Seco I 3134363738

Arrebola Bautista MA 22

Arrojo Agudo E 25

Arrojo Martín E 16

Arroyo J 1032

Arroyo MC 33

Arroyo R 16

Arsénio P 3437

Aru B 33

Ascaso Martorell J 26

Aseguinolaza C 15

Asensi A 134 TM2

Assali N 28

Ater M 212532

Ávarez Cobelas M 9

Ávila G 17

Azzioui O 24

Baccheta G 3336

Bachir Bouiadjra B 35

Baena L 27

Báez JC 2629

Bakkali Yakhlef S 35

Balao F 3133

Balbuena Gutiérrez E 26

Baldeón S 29

Bammi J 2729

Banabadji N 36

Bañares Baudet A 6

Bañares España E 3436

Barbara I 1237

Barbero M 15

Bariego Hernández P 2728

Barkat D 34

Barrajón Domenech G 33

Barrajón Mínguez J 33

Barrasa JM 36

Barreno E 4

Barrera I 11

Barros BSV 35

Bartoli A 32

Bartolo G 15

Basak UC 34

Basante Pol R 33

Bascones JC 7

Bastida F 1929

Bastos CJP 252930

Bayer E 13

Bazo Coronilla E 35

Becerra Parra M 262728303233343637

Belbachir N 35

Belkadi B 28

Bell PR 16 
Bello J 34

Beltrán H 29

Beltrán Tejera E 6

Ben Abdellah S 32

Ben El Mostafa S 26

Benabdelli K 3434

Benabid A 30

Benavente M 29

Benavente Navarro A 37

Benítez Cruz G 33

Benito Alonso JL 232425

Benlhabib O 28

Benmahioul B 35

Benmansour D 36

Berecibar E 37

Berjano R 28

Bermejo P 14

Bermejo R 3537

Bernardos S 2933

Berraho EB 32

Berrichi A 26

Betancort Villalba MJ 16

Bibiloni G 12

Biondi E 15

Blanca G 10111213141617323336

Blanco Dios JB 273337

Blanco Garrido F 35

Blanco Salas J 2627282933

Blasco Zumeta J 17

Bohorquez M 37

Boisset F 1114

Bootello M 141516

Borrego Aguayo F 32

Boyero JR 13

Brito KS 24

Brugués M 7

Brullo S 15

Burgarella C 32

Burgaz E 15

Butler I 29

Caballero G 2829303334

Cabello J 202335

Cabello ML 23

Cabezudo Artero B TM2 910111213141516

17181920212223242526272829303133

3435363738

Cáceres C 2728

Cacialli G 23

Calonge FD 66

Calvo L 15

Calvo MA 56

Cambra J 1417

Camiñas JA 26

Campos de la Cruz J 34

Camuñas E 23

Candau P 1420

Cano Carmona E 15182021222531

Cano L 13

Cano Maqueda J 2832

Cano Ortiz A 31
Cantero JJ 28

Cantó P 29

Cantos M 22

Cañadas Sánchez E 27

Capelo J 32

Carballal R 1627

Caron B 4

Caroti V 23

Carrasco MA 1327

Carratello A 23

Carreira JA 2028

Carrera D 34

Carretero JL 18

Carrillo López AF 212223242526

Carrillo P 16

Carrión FJ 1213

Carrión JS 21242628

Carrión Vilches MA 23242526

CasalsTortrás P 32

Casañ A 12

Casares M 71314

Casas de Puig C 711

Casasola Herrera JA 26

Casero Montes Z 34

Casimiro Soriguer F 333435363738

Casimiro Soriguer R 3133

Castelló Monsoriu AJ 2628

Castillo A 12

Castoldi E 35

Castro A 2933

Castro Antunes JH 36

Castro C 33

Castro J 1227

Castro ML 2732

Castroviejo S 30313132

Catalán P 3237

Cebolla Lozano C 38

Celis M 2022

Cerrillo MI 26

Chakrabarty D 32

Chamlal H 28

Chaparro AJ 32

Chaves A 27

Chenchouni H 37

Cires E 36

Cirujano S 2231

Clemente A 37

Cobo MD 31

Cobo Muro C 25

Cobos J 9

Coca B 28

Comas González A 34

Conde Álvarez RM 2234

Conde Poyales F TM2 91213141516171819

2021232526

Correia I 37

Cortés JE 2425

Costa JC 323437

Costa Talens M 15

Costa Tenorio M 1217 
Costas E 313438

Coy E 212223

Cremades J 12

Crespi AL 152933

Crespo A 4411

Crespo Villalba MB 121517192021222324

25262829

Cristóbal JC 2435

Cros RM 7

Cruz G 28

Cruz Pizarro L 16

Cuesta C 36

Cueto M 2728323335

Daguin F 35

Dakki M 29

Dana Sánchez ED 2326303134

Das SK 32

De Andrés Turrión ML 20

De la Fuente V 20

De la Rosa J 33343538

De la Torre Fernández F 25

De la Torre García A 131720212225272935

De las Heras MA 32

De Leonardis W 1419

De Paz Canuria E 23333435

De Sousa LMA 35

De Vega C 2828

Deil U 21222636

Del Palacio Izquierdo C 20

Del Río Sánchez J 36

Delgado Marzo JM 2635

Delgado Sánchez L 2628

Delgado Vázquez AJ 25283233

Descals E 13

Devesa Alcaraz JA 10131516172021232728

293031333334353636373838

Díaz Dapena J 31

Díaz de la Guardia C 10131617

Díaz Espejo A 23

Díaz González TE TM29 1011121314

Díaz J 35

Díaz Lifante Z 1920

Díaz Losada E 20

Díaz Such B 31

Díaz Tapia P 37

Diez Garretas B 1345 TM1 TM2

Díez MJ 192022262728

Diosdado JC 1819

Docampo S 2526

Doglio S 36

Dohou J 29

Domínguez Vilches E 916171832

Donaire F 30

Doncel Pérez E 2627

Dos Santos ACC 32

Dos Santos F 25

Dotor Fernández E 19

Douira A 2729

Doveri F 23

Dowell AB 212326
Draper D 283435

Dufour Dror JM 27

Dunabeitia MK 16

Ederra Indurain A 6

Egea JM 7101112131415192122

EgidoMazuelas F 3032

El Aisammi A 32

El Alaoui-Faris FE 24

El Houmaiz MA 36

El Khifli O 28

El Oualidi J 182122242527

Elena Rosselló JA 12

Elías Rivas MJ 31

Ennabili A 21

Ertas A 27

Escámez AM 13

Escribá Baeza MC 31

Escudero A 1517

Escudero M 3132

España Ramos L 911

Espinar JL 22

Espinar MC 13

Espirito Santo MD 153334

Esquivias R 37

Es-Sgaouri A 24

Esteve Chueca F 24

Esteve Reventós F 9

Estrada J 1324

Etayo J 1721

Fabregat Llueca C 2030

Fabregat M 28

Fagúndez J 2933

Fandos JV 31

Fanés Treviño 34

Fanlo Grasa E 32

Fanlo R 1

Farminhâo J 37

Félix LP 28

Felpete IA 27

Fennane M 25272933

Fenu G 33

Fernandes CP 33

Fernándes Lousa M 15

Fernández A 27

Fernández Areces MP 101214

Fernández C 37

Fernández Carrillo L 27

Fernández Carvajal MC 1436

Fernández Casado MA 36

Fernández de Castro C 37

Fernández Galiano E 81215

Fernández Gómez E 20

Fernández González D 24

Fernández González F 1315

Fernández I 1217192021222324

Fernández López C 9

Fernández Ordóñez MC 25

Fernández Pérez J 17

Fernández Prieto JA 1336

Fernández Rodríguez A 29 
Fernández S 23

Fernández Zamudio R 31

Ferrer Gallego PP 3031323435363738

Ferreras N 35

Figuerola R 1011

Filali Maltouf A 32

Fina A 14

Flagella M 38

Flores Moya A 1415171819202122232526

2931343638

Fraga Vila MI 9

Frances Causape MC 16

Freire Dopazo M 24

Freire L 6

Fuentes Carretero JM 38

Fuertes Lasala E 7

Fyad Lameche FZ 37

Galán C 32

Galán de Mera A 21222324252627282930

31313337

Galán R 9

Gallardo T 1113

Gallego Martín F 1218

Gallego MJ 9

Garbo Nadal R 36

García Adá R 17

García AI 131415

García B 29

García C 14

García Castaño JL 28

García de Nieto ME 16

García de Yébenes P 20

García Fernández ME 36

García Fuentes A 21252631

García González ME 1324292933

García I 15

García Manjón JL 6

García Martín F 111727

García ME 3335

García Molares A 162425

García Murillo P 2223303134

García Pausas J 32

García Pérez JC 6

García Prieto O 616

García R 14

García Río R 18

García RM 35

García Rojas JA 26273537

García Rowe J 11121323

García Ruíz R 28

García Salmones R 37

García Sanchez J 3138

García Sancho L 16

García Torres S 28

García V 37

García Verdugo JC 14

García Villada L 31

García Zamora P 24

Garg N 2526

Garrido Díaz B 2327
Garrido JA 2830

Garrido M 3536

Gaspar N 32

Gastón González A 27

Gavira O 2829303133343537

Géhu JM 3415

Géhu-Franck J 34

Ghezlaoui BE 36

Gibby M 8

Gil García JA 3 TM2 71012

Gil JM 10

Gil López MJ 36

Gil Sánchez L 1622

Gil Y 22232427

Giménez Luque E 262735

Giménez MA 5

Giraldez Fernández X 1119

Gmira M 29

Gómez Carrión J 26

Gómez Casero MT 32

Gómez D 15

Gómez García J 2

Gómez Garreta MA 529

Gómez Hernández P 142830

Gómez J 2938

Gómez Lama M 31

Gómez M 17

Gómez Mercado F 1215192627

Gómez Tejedor H 28

Gomis Blanco A 1733

Gómiz García F 303133

González A 31

González Ad 2728

González Bueno AI 121314151616171920

212326273335

González García JA 17181920

González Henríquez MN 16

González JA 16

González Jurado G 34

González Minero FJ 2022

González Porto AV 20

González Romano ML 1422

González Zapatero MA 12

Gonzalo R 30

Gradaille JL 14

Gros V 34

Gruber M 16

Guara Requena M 143031

Guarro J 56

Güemes J 192122

Guenoun I 35

Guerra J 3 TM1 TM2 79101113242628

Guerrero F 27

Guillén A 38

Guillot Ortiz D 28313334

Guirado J 2831

Guisande R 20

Guitían J 1315

Guitían P 13

Guma IR 3234 
Gupta N 34

Gutiérrez A 22

Gutiérrez Bustillo M 1416

Hafellner J 16

Haloui B 26

Hamamouch N 35

Hammada S 29

Hammoumi M 22

Haug H 22

Herbrard JP 16

Hernández Bermejo JE 1223

Hernández Cardona AM 112

Hernández Gallego Y 23

Hernández González A 212223242526

Hernández Hernández E 10

Hernández I 3537

Hernández Mariné MC 55

Hernández P 37

Herrera Grao A 35

Herrera J 17

Herrera M 13

Herrero B 22

Herrero Borgoñón JJ 12141729303233

Herrero Cembranos L 1315

Herrero Nieto A 172022

Hidalgo Fernández PJ 3237

Hidalgo Maqueda R 23

Hidalgo MI 141516192021

Hidalgo Triana N 3638

Hiraldo JA 22

Holttum RE 8

Honrubia M 51113

Horjales M 11

Iamonico D 34

Ibars A 121314

Ibn Tattou M 18262934

Idrissi Hassani M 29

Iglesias MI 182225

Infante F 17183234

Invernón VR 3438

Iranzo J 1011121314

Ivorra Jiménez A 3234

Izco J 131521293033

Izquierdo JL 1820

Jato Rodríguez MV 171822252627

Jiménez JF 2425

Jiménez López J 38

Jiménez Martínez JF 313637

Jiménez Mejías P 313234353738

Jiménez Merino C 34

Jiménez MN 1132

Jiménez Sánchez ML 2832

Juan A 2022242628

Juan R 20212223242627282930

Justo A 32

Kadiri M 36

Kaïd Harche M 35

Kazzaz M 232536

Kerdouh B 35

Khattabi A 29
Khelil B 35

Konaté I 32

Ladero Álvarez M 3715

Lado C 9

Laguna Lumbreras E 31323438

Lahlou H 32

Lahora Cano A 21303235

Lamela C 37

Lara Ruiz J 17

Larios JM 1113

Lavalle MC 30

Lázaro Bello JA 2728303336

Lázaro Gimeno D 34

Lazaro R 12

Lence C 34

Lendínez ML 34

Letreuch Belarouici A 3438

Letreuch Belarouici N 34

Liétor J 28

Linares JC 28

Linares JE 28

Linares Perea E 333437

Llamas F 12141527

Llamas Frade B 16

Lledó MD 21

Llimona X 57121422

Llorens L 1222

Loidi J 15

López Acosta P 31

López Albacete I 3235

López Blanco M 33

López E 3338

López Espinosa JA 31

López Fernández ML 6

López Figueroa F 34

López García D 37

López Ge 23

López González G 12133637

López J 30

López Martínez J 272835

López Martínez M 3032

López Nieto E 38

López Nieto JM 1516

López Pacheco MJ 10111314243237

López Pastora JA 32

López Rodas V 313438

López Tirado J 363738

López Udías S 2030

López Vélez G 19

López Vélez JP 16

Lora González A 2123

Lorite Moreno J 232530

Losa Quintana JM 3556

Lowe MR 25252636

Lucas Pimienta AB 272829

Lucena C 34

Luceño M 31323435

Lucía V 3638

Luis E 15

Luque B 26 
Luque T 14

Lyra GM 2632

Maarouf A 35

Maatougui A 36

Macías Fuentes FJ 25

Madamwar D 2526

Major A 8

Manoharan C 32

Manrique E 16

Marañon T 32

Marchal FM 34

Marchand C 22

Marcos N 13

Marín Murcia JP 35

Marques I 283435

Márquez AL 17

Martin A 2127

Martín Ballesteros MA 1920

Martín Bravo S 3237

Martín García JM 28

Martín Marcos MA 31

Martín Mosquero MA 21232627282930

Martínez Abaigar J 16

Martínez Arias R 29

Martínez de Azagra A 16

Martínez F 10

Martínez Hernández F 2830

Martínez JM 23

Martínez Ortega MM 192026

Martínez Parra JM 1215

Martínez R 33

Martínez Sagarra G 38

Martins A 33

Mascia F 36

Massjuk NP 27

Matarese Palmieri R 14

Mate Sadornil M 19

Mateo RG 35

Mateo Sanz G 9111214181920

Mateos Sanz MA 2834

Mateu I 1115

Mayor López M 3

Mediavilla Molina A 1718

Medina Cazorla JM 283031

Medina Jaramillo R 18

Medina L 32

Medjahdi B 3438

Mejías JA 19

Melendo Luque M 202125

Melgar Rueda M 32

Melhem T 25

Méndez J 2525

Mendoza AJ 3032

Menezes de Sequeira M 31

Merinero S 36

Merzouk A 36

Mesa Coello R 38

Miedes Marqués E 36

Miguel Alonso A 17

Minervino Netto A 2426
Minissale $\mathrm{P} 15$

Mishra S 34

Moglia MM 2631

Molero Mesa J 111316

Molina A 131515

Molina Abril JA 152326333435

Molina Cantos R 22

Monasterio Huelin E 18

Monsó Senabre MA 16

Montegaudo FJ 29

Monteiro Henriques T 323437

Montenegro G 17

Montero A 36

Montilla RJ 31

Montserrat G 15

Montserrat P 1

Morales Alonso R 242931

Morales C 14

Morales del Molino C 35

Morales Valverde R 313436

Moreno A 25

Moreno D 2831

Moreno Durán MD 22

Moreno G 234691113

Moreno M 1117

Moreno PP 121415

Moreno Robledo C 33

Moreno Socías E 19

Moret Besanet A 1616

Morinho J 37

Morla Juaristi C 18

Mota Poveda JF 1215192023262728303234

Müller J 26

Muñoz Álvarez JM 9102325263137

Muñoz AR 33

Muñoz Centeno LM 2728

Muñoz Colomina I 18

Muñoz Rodríguez AF 171825313235

Muñoz Secilla F 37

Muralitharan G 32

Muthukumar C 32

Nadal Puigdefabregas M 16

Narbona E 28

Nascimento OS 38

Navarro Aranda C 716

Navarro C 14

Navarro F 12

Navarro G 26

Navarro Reyes FB 23273236

Navarro T 151617181920212223242633

Navas Fernández D 22232427282930

Navas Fernández P 22232427

Nebot M 22

Neto C 323437

Niell FX 20

Nieto Caldera JM TM2912131415161718

19202234

Nieto Gil I 31

Nieto RM 13

Nitiu DS 34 
Nogueira I 1316

Noguerol Seoane A 5516

Noya R 16

Nunes JMC 22232425263238

Núñez C 28

Ocaña E 21

Ojeda Land E 38

Ojeda Nempu P 1823

Oliva R 71314

Oliver X 32

Oltra B 31

Oltra Benavent JE 37

Oria de Rueda Salguero JA 16

Ormonde J 1316

Orriach R 3334

Orshan G 17

Ortas L 38

Ortega A 692528

Ortega González F 2734

Ortega JM 22

Ortega Olivencia A 141415161727282931

Ortiz D 38

Ortiz Herrera MA 2833

Ortiz PL 1719192028

Ortiz S 12131421

Ortúñez Rubio E 20

Otálora MAG 36

Ouhammou A 34

Ouyahya A 212429

Oyonarte C 20

Pacheco J 15

Padrón Mederos MA 323438

Paiva Lima LF 34

Pajarón Sotomayor S 141720

Palacios González M 2334

Panneerselvam A 32

Pardo de Santallana M 29

Parra G 27

Parra R 20

Passarelli LM 3233

Pastor JE 141718192021222324262728 2930

Paula ÉJ 2526

Pavón M 353638

Payá Mira J 2225

Paz Canuria E 2430

Pazo JP 5

Pedreira ES 26

Pedreira FA 38

Pedreño JG 12

Pedrocchi C 23

Pedrol Solanes J 26

Peinado Lorca M 91215

Pena Martín C 29

Penas Merino A 910111314152324

Peña V 37

Peñas de Giles J 202331

Pereira AA 33

Pereira AJ 3637

Pereira da Silva HSV 35
Pereña Ortiz J 3234

Pérez Badía R 15

Pérez Carro FJ 101214

Pérez Chiscano JL 71535

Pérez Contreras J 3637

Pérez García FJ 283234

Pérez Hornero MJ 1920

Pérez Jarauta T 16

Pérez Latorre AV 13141516171819202122

23242525262728293031333435363738

Pérez Llorens JL 3537

Pérez Losantos A 6

Pérez Morales C 131415

Pérez Porras C 27

Pérez Raya F 11131516

Pérez Rubín J 273435353637

Pérez Sánchez FJ 31

Pérez Santigosa N 34

Pérez Sanz S 12

Pericás JJ 11

Peris JB 38

Peteiro C 37

Petryna L 28

Philips I 26

Pichi-Sermolli REG 16

Piera J 2225

Pina FJ 28

Pinar García S 19

Pinilla R 23

Pinto Cruz C 333437

Pinto D 36

Pisco García JM 18

Pizarro J 1331

Plaza Arregi L 3237

Plazuelo A 34

Polo Alberti L 5

Portela Pereira E 37

Porto M 3637

Posadas Fernández L 32

Posudin YI 27

Prada C 81122293133

Prados Ligero J 23

Prats Fonts S 32

Prefasi M 19

Prelli R 38

Prunier F 35

Puche F 91012

Puech S 18

Puente García E 1011131523243032

Pujadas Salvà AJ 12212225303132333436 3738

Pulgar I 2130

Queiros M 1316

Quesada Rincón J 3334

Quezel P 15

Quijada J 25

Quintanar A 32

Rahou A 27

Rahouti M 32

Ramalho CE 36 
Ramil Rego P 1518

Ramírez García C 1823

Ramírez González JM 34

Ramírez J 55

Ramírez López J 3234

Ramos A 11

Ramos I 11

Ramos Maqueda S 2627282930

Real R 29

Recio M 1920212223242526

Redondo N 11

Rego R 37

Reichstein T 8

Reinoso Franco J 10121617181921232538

Rendón Martos M 34

Rendón Vega JL 33

Renobales G 1629

Rentería J 5

Rey Bueno MM 22

Reyes Betancort JA 323438

Reyes G 2020

Reyes Sepúlveda E 32

Rhazi L 35

Riadi H 23253536

Ribera Siguan MA 5

Richardson M 37

Rico Hernández E 11192026283136

Rico JM 37

Rico R 131415

Rico VJ 1516

Riera Fernández P 37

Riera J 29

Rigual A 28

Rios GI 38

Ríos Ruiz S 18192021

Rita J 1214

Rivas Martínez S 234 TM2 81113

Rivas Rangel A 32

Rivera González MC 36

Rivera J 10

Robledo Miras A 181920

Robles AB 27

Robles Domínguez E 3233343637

Rocha Afonso ML 8

Rocha J 33

Rocha JRS 3335

Rochdi M 30

Rodíguez Rajo FJ 252627

Rodrigues EP 35

Rodríguez de los Santos M 22

Rodríguez E 35

Rodríguez González PM 35

Rodríguez Hiraldo C 30

Rodríguez Marzal JL 24

Rodríguez Nozal R 16171819202122232627

Rodríguez Oubiña J 121421

Rodríguez Ramos N 32

Rodríguez Riaño T 27

Rodríguez Tamayo ML 28

Roldán A 1316
Rolleri C 293133

Román del Castillo B 32

Roman JM 17

Romaris XM 5

Romero García AT 14161732

Romero Rodríguez C 6

Romero T 1136

Romero Zarco C 101829313334353738

Romeu M 37

Romo A 15

Ros M 16

Ros RM 911132428

Rosa MV 27

Roselló R 38

Rosselló Graell A 2834

Rosselló JA 111214

Rossito M 14

Rosua JL 11121516

Rovira Forcada SJ 16

Rozas Espada A 28

Rubiales Olmedo D 3032

Rubio Sánchez A 1215

Rudner M 36

Ruiz de Clavijo E 9101116253436

Ruíz de Gopegui A 343537

Ruiz Girela M 3038

Ruiz Téllez T 13162334

Ruiz Y 37

Rupidera Giraldo JL 16

Saa Otero PM 1520

Sáez Ll 14181923

Sáez Soto F 19

Salazar Mendías C 21252627323334

Salgueiro FJ 2328

Salhi G 36

Salinas Bonillo MJ 1635

Salvador Nemoz ML 16

Salvadores R 37

Salvo Tierra AE 5 TM1 TM2 791011121314

151722

San Martín Padovani C 1823

Sánches del Río J 31

Sánchez A 19202021

Sánchez Agudo JA 26

Sánchez Almendro AJ 2526

Sánchez Anta MA 1218

Sánchez Casimiro Soriguer C 38

Sánchez Castillo PM 13163436

Sánchez Crespo A 4

Sánchez García I 2021212527

Sánchez Gómez P 12192122232425263135

3637

Sánchez Gullón E 24252627293031323335

Sánchez Lorencio MI 36

Sánchez Mata D 2326

Sánchez N 37

Sánchez Pascual NR 1821

Sánchez Pedraja O 31

Sánchez Rodríguez JA 111631

Sánchez Tundidor LF 2527 
Sancho LG 16

Santa Bárbara C 2228

Santos ACC 2426

Santos GN 38

Santos Guerra A 323438

Santos MT 15

Santos R 37

Santos Vicente M 2831

Sanz Elorza M 2632

Sanz Fábrega F 12

Saoud Y 36

Saraiva S 37

Sarasa Alcubierre N 2628

Sardinero S 23

Sastre B 14

Scholz H 24

Secilla A 37

Segarra Moragues JG 2426

Seigle Murandi F 32

Seijó Coello MC 17222426

Selma C 13

Seoane Camba J 5

Sergio C 71134

Seriña E 16

Seriñá E 16

Serra L 2022

Serra M 12

Serrano M 34

Shah V 2526

Sharma H 28

Sierra de Cózar G 32

Silva I 25

Silva Pando FJ 12

Silva V 333437

Silvestre S 17

Simo RM 7

Singh P 33

Singh SM 33

Sleep A 8

Smythies BE 2

Snow N 35

Sobrino Vesperinas E 2632

Socorro Abreu O 1113

Sola AJ 2832

Solanas Ferrándiz JL 2022

Soler MR 24

Sonnentag M 24

Soñora FX 13141718

Soria JM 16

Soria P 32

Soriano P 1527

Soto Moreno J 1112131416192021

Sousa Martín A 31

Souza Egypsi V 35

Spampinato G 15

Stuessy T 28

Suárez Cervera M 12

Suárez Santiago VN 273436

Suau R 131415

Tahiri H 24
Talavera Lozano S 172023283131323338

Talavera Solís M 313338

Taleb MH 33

Tamajón R 23

Tarrega I 11

Tarrega R 15

Tauleigne Gomes C 27353637

Tavares AM 37

Tavira J 25

Tébar FJ 22

Telleria MC 20

Telleria MT 6

Terrab Benjelloun A 262728313338

TerrobaValadés J 32

Terrón Alfonso A 16

Thajuddin N 3233

Thulin M 13

Tison JM 32

Tormo R 1525

Toro FJ 19202122232425

Torres Cordero JA 212527

Torres J 14

Torres N 11

Tortosa RD 32

Tremetsberger K 28

TrianoMunoz E 323437

Trigo MM 131415161718192021222324 25263134

Troncoso A 22

Ubera JL 9

Uribe P 15

Valdés Bermejo E 241228

Valdés Castrillón B 1922262728

Valdés Franzi A 22

Valeiras J 26

Valencia Barrera RM 24

Valle CJ 15

Valle Tendero F 10121518202123252633

Valtueña MJ 31

Van der Meer P 2834

Vaquero de la Cruz J 17

Vargas JM 29

Varo J 3557

Vasconcelos JNC 38

Vázquez Pardo FM 151621242627282930

3133

Vázquez VM 4

Vega Maray A 24

Vera González JJ 1415

Vera Pérez JB 263136

Vergara JJ 3537

Verloove F 33

Vicedo M 2122

Vicens J 141418

Vicente A 35

Vicente E 6

Vicente Orellana JA 2122232431333437

Vida G 8

Viera Benítez MC 1316171819212338

Vijayakumar R 32 
Vilar L 1519

Vilar M 1011121314

Vilas Bôas-Bastos B 2529

Villar JL 35

Villar L 115

Villarrubia MJ 17

Viñas X 1519

Vitorino JL 37

Vizoso MT 1627

Weickert P 293132

Wildpret de la Torre W 6

Yammi K 29
Yano O 2930

Yera J 38

Youcef Benkada M 35

Yus Ramos R 31

Zafra ML 11

Zamora JC 38

Zanolla M 3538

Zárate R 22

Zea MM 16

Ziri R 29

Zizza A 1419

\section{ANEXO II NOVEDADES TAXONÓMICAS Y VOLUMEN DONDE SE CITAN}

Alyssum granatense Boiss. et Reuter var longipetalum

P. Montserrat

Andryala x brievaensis García Adá

Anthyllis plumosa E. Domínguez

Anthyllis $x$ fortuita M. Guara \& P. P. Ferrer

Armeria $x$ carnotana Blanco-Dios

Armeria $x$ cintrana Tauleigne Gomes

Arrhenatherum album (Vahl) W. D. Clayton var. erianthum (Boiss. \& Reuter) Romero Zarco $\mathbf{1 0}$

Arrhenatherum elatius (L.) Beauv. subsp. baeticum Romero Zarco

10

Asplenium marinum L. var. depauperatum Diaz \&

Salvo fma. xerophilum (Merino) T.E. Diaz \& A.E.

Salvo

TM2

Asplenium marinum L. var. depauperatum T.E. Diaz \& A.E. Salvo

TM2

Asplenium marinum L. var. marinum fma. incisum (Christ) T.E. Díaz \& A.E. Salvo

TM2

Asplenium marinum L. var. praealtum T.E. Diaz \& A.E. Salvo

TM2

Avenula marginata (Love) J. Holub subsp. albinervis (Boiss.) Romero Zarco

18

Avenula marginata (Love) J. Holub subsp. cintrana (Róser) Romero Zarco

18

Avenula marginata (Love) J. Holub subsp. gaditana (Romero Zarco) Romero Zarco

18

Avenula marginata (Love) J. Holub subsp. marginata var. reuteri (Romero Zarco) Romero Zarco

18

Avenula sulcata (Gay ex Delastre) Dumort. subsp. albinervis (Boiss.) Rivas Martínez

2

Baldellia repens (Lam.) Ooststroom subsp. baetica

Talavera \& R. Casimiro-Soriguer

33

Baldellia repens (Lam.) Ooststroom subsp. cavanillesii (Molina-Abril et al.) Talavera 33

Beta maritima subsp. marcosii (O. Bolós \& Vigo) A. Juan \& M.B. Crespo

24

Blechnum fernandezianum (Looser) Prada \& Rolleri 31

Blechnum mochaenum G. Kunkel subsp. achalense
(Hieron.) Prada \& Rolleri

Blechnum mochaenum subsp. squamipes (Hieron. )

Prada \& Rolleri

31

Campanula cabezudoi Cano-Maqueda \& Talavera

Campanula rotundifolia L. subsp. willkommii

(Witasek) Blanca 32

Carduncellus hispanicus Boiss. subsp. intercedens

(Degen \& Hervier) G. López

37

Carduncellus monspelliensium All. var. almeriensis

G. López

37

Carduncellus monspelliensium All. var. valentinus

G. López

37

Carduncellus rhiphaeus (Font Quer \& Pau)

G.López

37

Carduncellus sect. Thamnacanthus (DC.) G.

López

37

Carduncellus ser. Fruticosi G. López $\quad 37$

Carex castroviejoi Luceño \& Jiménez Mejías 34

Carlina corymbosa L. subsp. major (Lange) J. López

\& Devesa

35

Centaurea boissieri DC. subsp. integrifolia (Willk.)

Blanca \& Suár.-Sant. $\quad 36$

Centaurea castellanoides Talavera subsp. arundana

E. López \& Devesa

33

Centaurea castellanoides Talavera subsp. talaverae

E. López \& Devesa

Centaurea gallaecica (Laínz) Arnelas \& Devesa37

Centaurea graminifolia (Lam.) Muñoz Rodr. \&

Devesa

35

Centaurea nigra subsp. endressii (Dostál) Arnelas

\& Devesa

36

Centaurea susannae Invernón \& Devesa var. paivae

Invernón \& Devesa

38

Centaurea triumfettiii All. subsp. semidecurrens

(Jord.) Dostál var. semidecurrens (Jord.) Muñoz

Rodr. \& Devesa

Centaurea ultreiae Silva Pando var. bazarensis

Blanco-Dios

Centranthus lecoqii var. longecalcaratus (Pau) 
López \& Devesa

Cladanthus mixtus (L.)Chev. var. aureus (Durieu) Gómiz

33

Crepis bermejana M. Talavera, C. Sánchez Casimiro-

Soriguer \& S. Talavera

38

Crepis hyemalis (Biv.) M. Talavera

38

Crepis stellata (Ball) M. Talavera

38

Cytisus malacitanus Boiss. subsp. moleroi (Fern.

Casas) A. Lora, Hero. Berm. \& J. Prados 23

Echinospartum algibicum Talavera \& Aparicio 20

Endopappus macrocarpus Sch. Bip. subsp. macrocarpus var. aureus (L. Chevall.) Gómiz 33

Erigeron cabelloi A. Pujadas, R. García-Salmones \& E. López

37

Eunotia pseudopectinalis Hustedt var. vasconiensis (Manguin) Sánchez Castillo

13

Fedia cornucopiae var. scorpioides (Dufresne) López \& Devesa

28

Galium aparine L. subsp spurium (L.) Hartman var. aparinella (Lange) Ortega-Olivencia \& Devesa

28

Galium estebani var. leioclados (Pau) OrtegaOlivencia \& Devesa

28

Galium papillosum Lapeyr. subsp. helodes (Hoffmanns. \& Link) Malag. ex Ortega-Olivencia \& Devesa

Galium saxatile var. vivianum (Kliphuis) OrtegaOlivencia \& Devesa

Gastridium lainzii (Romero García) Romero Zarco

Genista longipes Pau subsp. viciosoi Talavera \& Cabezudo

Genista pumila (Debeaux \& E. Rever. ex Hervier) Vierh. subsp. rigidissima (Vierh.) Talavera \& LI. Sáez

Gentiana angustifolia Vill. subsp. corbariensis (Braun-Blanq.) Renob.

Helictochloa Romero Zarco sect. Pratavenastrum (Vierh.) Romero Zarco

Helictochloa Romero Zarco sect. Scleravenastrum (Holub)Romero Zarco

Holcus annuus C. A. Mey. subsp. setiger (Nees) M. Seq. \& Castrov

Holcus annuus C. A. Mey. subsp. setiglumis (Boiss. \& Reut.) M. Seq. \& Castrov.

Holcus lanatus L. subsp. tuberosus (Salzm. ex Trin.) M. Seq. \& Castrov.

Holcus lanatus L. subsp. vaginatus (Willk. ex Perez Lara) M. Seq. \& Castrov.

Hormathophylla lapeyrousiana (Jordan) Kiipfer subsp. angustifolia (Willk.) Rivas Martínez 2 Jasonia bocconei (Brullo) Pardo de Santayana \& Morales

Jasonia lopadusanus (Brullo) Pardo de Santayana \& Morales

Juncus tenageia var. perpusillus (Fern.-Carv. \& F. Navarro) Romero Zarco
Knautia nevadensis var. lanceolata (Pau) Devesa, Ortega-Olivencia \& López $\mathbf{2 8}$

Knautia numantina (Pau) Devesa, Ortega-Olivencia \& López

28

Knautia rupicola var. macrotrycha (Pau ex Font Quer) Devesa, Ortega-Olivencia \& López $\mathbf{2 8}$

Knautia subscaposa var. alboi (Sennen) Devesa, Ortega-Olivencia \& López $\quad 28$

Koeleria crassipes Lange subsp. filifolia (Domin) Quintanar \& A. T. Romero 32

Koeleria dasyphylla Willk. subsp. nevadensis (Hackel) Quintanar \& A. T. Romero 32

Lamium gevorense (Gómez Hernández) Gómez Hernández \& A. Pujadas 30

Limonium malacitanum Díez-Garretas TM2

Lomelosia graminifolia var. virescens (Jord. \& Fourr.) Devesa, Ortega-Olivencia \& López $\mathbf{2 8}$

Macrochloa antiatlantica (Barreña, D. Rivera, Alcaraz \& Obón) F. M. Vázquez 31

Macrochloa tenacissima (Loefl. ex L.) Kunth subsp. umbrosa F.M. Vázquez 31

Marasmius litoralis Quél. var. ventallonii (Sing.) Moreno

Moricandia moricandioides subsp. pseudofoetida Sánchez Gómez, Carrrión Vilches, Hernandez \& J. Guerra

26

Nepeta amethystina Poiret in Lam. subsp. anticaria Cabezudo, Nieto Caldera et Navarro $\quad \mathbf{1 6}$

Ononis cuatrecasasii Devesa 21

Ononis gines-lopezii Devesa 21

Onopordum hinojense S. Talavera, F. Balao, R. Casimiro-Soriguer, M. Talavera Solís, A. Terrab et M.A. Ortiz

33

Onopordum magrebiense S. Talavera, F. Balao, R. Casimiro-Soriguer, M. Talavera Solís, A. Terrab et M.A. Ortiz

33

Ophrys scolopax $\times$ Ophrys speculum $\quad 37$

Orobanche ballotae A. Pujadas $\mathbf{2 2}$

Orobanche densiflora Salzm. var. tarifae A. Pujadas $\mathbf{3 3}$

Orobanche lainzii (J. Gómez Navarro et al.) Triano \& A. Pujadas 37

Orobanche mariana A. Pujadas $\quad 32$

Orobanche mutelii F. W. Schultz subsp. reuteri (Carlón, Gómez, Laínz, Moreno, Sánchez \& Schneeweiss) A. Pujadas 32

Orobanche subgen. Boulardia (F.W. Schultz) A. Pujadas

32

Orthotrichum cupulatum Brid. subsp. baldaciii (Bott. \& Vent.) Guerra 10

Peucedanum officinale L. subsp. brachyradium García-Martín et Silvestre 17

Phlomis crinita Cav. subsp. malacitana (Pau) Cabezudo, Nieto Caldera et Navarro 16

Phlomis italica L. subsp. antiatlantica (Peltier) Rivas Martínez

Phlomis purpurea L. subsp. almeriensis (Pau) Losa 
\& Rivas Goday

Phlomis purpurea L. subsp. caballeroi (Pau) Rivas Martínez

\section{2}

Phlomis $x$ composita Pau nm. almijarensis (Pau) Mateu

Phlomis $x$ composita Pau nm. malacitana (Pau) Mateu

Phlomis x composita Pau nm. trullenquei (Pau) Mateu

Physalis philadelphica Lam. subsp. ixocarpa (Brot. ex Hornem.) Sobrino-Vesperinas \& Sanz-Elorza

Poa trivialis L. subsp. feratiana (Boiss. et Reuter) Hernández Cardona

\section{2}

Salix x coenocarpetana Rivas-Martínez, T.E. Díaz, J.A. Prieto, J. Loidi \& A. Penas

Salix x expectata Rivas-Martínez, T.E. Díaz, J.A.

Prieto, J. Loidi \& A. Penas

Salix $x$ longissima T.E. Díaz \& J. Andrés $\mathbf{1 2}$

Salix $x$ multidentata T.E. Díaz \& F. Llamas $\mathbf{1 2}$

Salix x pormensis T.E. Díaz \& F. Llamas $\quad 12$

Salix x pseudoelaeagnos T.E. Díaz \& F. Llama $\mathbf{1 2}$

Salix x pseudosalvifolia T.E. Díaz \& E. Puente $\mathbf{1 2}$

Salix x rijosa Rivas-Martínez, T.E. Díaz, J.A.F.

Prieto, J. Loidi \& A. Penas

12

Salix $x$ viridifolia T.E. Díaz \& A. Penas $\quad 12$

Salvia lavandulifolia Vahl. subsp. amethystea

(Emberger \& Maire) Rosúa \& Blanca

11

Salvia lavandulifolia Vahl. subsp. blancoana (Webb

\& Heldr. ) Rosúa \& Blanca

11

Salvia lavandulifolia Vahl. subsp. blancoana (Webb

\& Heldr. ) Rosúa \& Blanca var. aurasiaca (Maire)

Rosúa \& Blanca

Salvia lavandulifolia Vahl. subsp. maurorum (Ball)

Rosúa \& Blanca

Salvia lavandulifolia Vahl. subsp. mesatlantica

(Maire) Rosúa \& Blanca

Salvia lavandulifolia Vahl. subsp. vellerea (Cuatr.) Rivas Goday \& Rivas Martínez var. lagascana (Webb) Rosúa \& Blanca

Sarcocapnos baetica (Boiss. \& Reuter) Nyman subsp. ardalii López Vélez

Satureja obovata Lag. subsp. hispalensis (Pau)

Cabezudo, Nieto Caldera et Navarro

Satureja obovata Lag. subsp. malacitana (G. López)

Cabezudo, Nieto Caldera et Navarro $\quad 16$

Satureja obovata Lag. subsp. valentina (G. López)

M. B. Crespo

Sideritis chamaedryfolia Cav. subsp. littoralis M.B.

Crespo, Solanas, De la Torre et Payá 25

Sideritis flavovirens (Rouy) Alcaraz, Peinado, Martínez-Parra, Carrión \& Sánchez-Gómez 12

Sideritis funkiana Willk. subsp. talaverana Socorro. Cano \& Espinar

Sideritis granatensis (Pau) Alcaraz, Peinado,

Martínez-Parra, Carrión \& Sánchez-Gómez 12

Sideritis hirsuta L. subsp. danielii (Rivera \& Obón)
Bernardos, Aguiar \& Amich

29

Sideritis incana L. subsp. occidentalis (Font Quer)

Cabezudo, Nieto Caldera et Navarro $\quad \mathbf{1 6}$

Sideritis leucantha Cav. subsp. bourgeana (Boissier)

Alcaraz, Peinado, Martínez-Parra, Carrión \&

Sánchez-Gómez

12

Sideritis leucantha Cav. subsp. incana (Willk.) Alcaraz, Peinado, Martínez-Parra, Carrión \&

Sánchez-Gómez

12

Sideritis osteoxylla (Pau) Alcaraz, Peinado, Martínez-Parra, Carrión \& Sánchez-Gómez 12

Silene gazulensis Galán de Mera, Cortés, Vicente Orellana \& Morales Alonso $\quad 24$

Stipa almeriensis F.M. Vázquez $\quad \mathbf{3 1}$

Stipa apertifolia Martinosvky var. nevadensis

Vázquez \& Devesa 21

Stipa bufensi F.M. Vázquez, H. Scholz \& M. Sonnentag 24

Stipa cazorlensis (F.M. Vázquez \& Devesa) F.M.

Vázquez, H. Scholz \& M. Sonnentag 24

Stipa filabrensis H. Scholz, M. Sonnentag \& F.M. Vázquez 24

Stipa gigantea Link . subsp. donyanae Vázquez \& Devesa

Stipa iberica Martinovsky var. austro-iberica

(H.Scholz) Vázquez \& Devesa 21

Stipa iberica Martinovsky var. pseudodasyphylla

(Martinovsky) Vázquez \& Devesa

21

Stipa juncea L. var. cabanasii Vázquez \& Devesa

Stipa parviflora Desf var. pilosa (Chrtek \& Martinovsky) Vázquez \& Devesa 21

Stipa pauneroana (Martinovsky) Vázquez \& Devesa 21

Taeniatherum caput-medusae (L.) Nevski var. asperum (Simonk.) A. Pujadas $\quad \mathbf{3 8}$

Teucrium $\times$ motae Lahora \& Sánchez Gómez $\mathbf{3 5}$ Teucrium $\times$ pierae Gómez Nav., P.P. Ferrer, R. Roselló, A. Guillén, E. Laguna \& J.B. Peris 38 Teucrium ambiguum (Lge.) Esteve Chueca 2 Teucrium capitatum L. subsp. gracillimum (Rouy) Valdés-Bermejo

Teucrium edetanum M.B. Crespo, Mateo \& T.Navarro

Teucrium embergeri (Sauvage \& Vindt) El Oualidi,

T. Navarro \& A. Martin 22

Teucrium joannis (Sauvage \& Vindt) El Oualidi, T. Navarro \& A. Martin 22

Teucrium lusitanicum Schreber, subsp. aureoformis (Rouy) Valdés-Bermejo

Teucrium mesanidum (Litard \& Maire) Navarro y Rosúa

Teucrium mitecum M. Ibn Tattou \& J. El Oualidi18 Teucrium rifanum (Maire \& Sennen) T. Navarro \& El Oualidi

Teucrium soloitanum (Maire) Navarro y Rosúa 16 Teucrium x carvalhoi A. F. Carrillo, A Hernández, 
E. Coy, Gtiemes \& Sánchez-Gómez

Teucrium $x$ conquense M.B. Crespo \& Mateo nothosubsp. siyasense Carrillo \& Sánchez Gómez

21

Teucrium x guemesii J.F. Jiménez, Carrillo, Carrión

Vilches, P. Sánchez-Gómez \& T. Navarro $\quad 24$

Teucrium x navarroi Sánchez Gómez, Güemes \& Carrillo

21

Teucrium x portusmagnii P. Sánchez Gómez, Carrillo, A. Hernández \& T. Navarro

24

Teucrium x pseudothymifolium Sánchez Gómez,

Güemes \& Carrillo

21

Teucrium $x$ rigualii De la Torre et Alcaraz

17

Teucrium $x$ riosii De la Torre et Alcaraz

Teucrium $x$ riverae De la Torre et Alcaraz

Teucrium $x$ robledoi De la Torre et Alcaraz

Thymus $\times$ ahimae M.B. Crespo, M.A. Alonso \& J.

Riera

Thymus $x$ herberoi De la Torre, Vicedo, Alonso \& Paya
Thymus $x$ viteki $\mathrm{R}$. Morales

Trifolium L. sect. trifolium subsect. diffusa A. Muñoz

Trifolium phleoides Pourret ex Willd. subsp. willkommii (Chab.) A. Muñoz

17

Trifolium striatum L. subsp. brevidens (Lange) A. Muñoz

Ulex baeticus Boiss. subsp. bourgaeanus (Webb)

Pérez Latorre \& Cabezudo

23

Ulex rivasgodayanus (Cubas) Cabezudo y Pérez Latorre

28

Valeraniella locusta (L.) Laterrade subsp. locusta f. carinata (Loisel.) Devesa, J.López \& R. Gonzalo

30

Valeraniella coronata (L.) DC. in Lam. \& DC f. pumila (L.) Devesa, J. López \& R. Gonzalo 30 Valeraniella dentata (L.) Pollich f. rimosa (Bastard) Devesa, J. López \& R. Gonzalo 30

Valeraniella multidentata Loscos \& J. Pardo. var. oscensis (Fanlo) Devesa, J. López \& R. Gonzalo

\section{ANEXO III \\ NOVEDADES SINTAXONÓMICAS Y VOLUMEN DONDE SE CITAN}

Aceri obtusati-Ostryetum carpinifoliae subas. ostryetosum carpinifoliae

15

Aceri obtusati-Ostryetum carpinifoliae subas. quercetosum congestae

15

Aceri obtusati-Ostryetum carpinifoliae subas. tilietosum platyphylli

15

Santolinion pectinato-canescentis subas. Andryalo ragusinae-Artemisietum barrelieri

Suaedo braun-blanquetii-Tamaricetum boveanae

Abieto pinsapo-Juniperetum phoeniceae

Abieto pinsapo-Juniperetum phoeniceae subas. pinetosum halepensis

24

Abieto pinsapo-Juniperetum phoeniceae subas. rhamnetosum oleoidis

24

Abieto pinsapo-Juniperetum sabinae

23

Acacio macracanthae-Tecometum guarumis $\mathbf{3 4}$

Acarosporetum heuflerianae subas. caloplacetosum teicholytae

Adenocarpion argyrophylli

Adiantetalia raddiani

Adiantetum subvolubilis

Adiantion subvolubilis

Agrostio stoloniferae-Tamaricetum canariensis subas. inuletosum crithmoidis

Alismo-Sagittarietum montevidensis

Alnetalia acuminatae

Anacyclo radiati-Hordeetum leporini subas. centaureetosum sonchifoliae

Androsacetum pyrenaicae

Andryalo ragusinae-Artemisietum barrelieri
Andryalo ragusinae-Artemisietum barrelieri subas. salsoletosum genistioidis

12

Andryalo ragusinae-Artemisietum barrelieri subas. santolinetosum canescenti

12

Anthyllido plumosae-Andryaletum ramossisimae 29

Anthyllido plumosae-Andryaletum ramossisimae subas. centranthetosum nevadensis

29

Anthyllido plumosae-Andryaletum ramossisimae subas. moehringietosum tejedensis $\quad 29$

Anthyllido-Arenariatum alfacarensis $\mathbf{1 5}$

Anthyllido-Cistetum clusii subas. ononidetosum edentulae $\mathbf{1 0}$

Antirrhino cirrhigeri-Parietarietum judaicae $\mathbf{2 5}$

Antirrhino cirrhigeri-Parietarietum judaicae subas. cymbalarietosum muralis

25

Aquilegio nevadensis-Gymnocarpietum robertiani

31

Arbolo unedonis-Quercetum pyrenaicae subas. blechnetosum spicantis

Arbuto unedonis-Quercetum pyrenaicae subas. quercetosum suberis

33

Arenarietum arundanae

23

Arenario (retusae)_Linarietum salzmannii 4

Arenario conimbricensis-Sedetum mariani $\mathbf{3 6}$

Aristeguietio discoloris-Baccharidetum latifoliae

Aristeguietio discoloris-Baccharidetum latifoliae subas. polylepidetosum racemosae $\quad 29$

Aristeguietio discoloris-Baccharidetum latifoliae subas.proustietosum berberidifoliae $\quad \mathbf{2 9}$

Aristido adscensionis-Chloridion virgatae $\quad \mathbf{2 7}$ 
Armatocereo riomajensis-Euphorbietum apurimacensis

34 Armerio francoi-Arrhenatheretum sardoi $\quad 33$

Artemisio barrelieri-Salsoletum genistioidis 12 Artemisio glutinosae-Santolinetum semidentatae11 Arthrocnemo glauci-Salicornietum emerici 4 Arthrocnemo glauci - Salicornietum emerici subas. typicum

Arthrocnemo glauci - Salicornietum ramosissimae subas. salicornietosum emerici

Arthrocnemo glauci - Salicornietum ramosissimae subas. Typicum

4

Arthrocnemo glauci-Salicornietum emerici subas. salicornietosum ramosissimae

4

Arthrocnemo glauci-Salicornietum ramosissimae4

Asparago albi-Rhamnetum oleoidis subas. ceratonietosum siliquae

24

Asparago albi-Rhamnetum oleoidis subas. ericetosum scopariae

18

Asparago aphylli-Calicotometum villosae subas. Ephedretosum fragilis

25

Asparago aphylli-Calicotometum villosae subas. genistetosum linifoliae

18

Asparago aphylli-Calicotometum villosae subas. juniperetosum turbinatae

21

Asparago horridi-Juniperetum turbinatae subas. ceratonietosum siliquae

38

Asperulo hirsutae-Ulicetum scabri subas. anthyllidetosum cytisoidis

21

Asplenietum hispanici

Asplenio billotii-Dianthetum lusitani

13

Astragalo nevadensis-Bupleuretum spinosi

23

Atriplici roseae-Salsoletum ruthenicae

23

Baccharidetalia salicifoliae

Baccharido salicifoliae-Gynerietum sagittati 29

Baccharido tricuneatae-Puyetum raimondii $\quad 34$

Bacopo myriophylloidis-Eleocharitetum minimae

33

Balloto hirsutae-Lavateretum maritimae subas. salsoletosum oppositifoliae

33

Barnadesio blakeanae-Ophryosporidetum peruviani

Belloo schultzii-Salpichroion glandulosae $\quad \mathbf{2 8}$

Beloo piptolepis-Dissanthelietutn calycini $\mathbf{2 8}$

Berberido hispanicae-Quercetum rotundifoliae subas. quercetosum fagineae

15

Beto marcosii-Medicaginetum citrinae

24

Beto marcosi-Medicaginetum citrinae subas. salsoletosum oppositifoliae

Biscutello baeticae-Anthoxanthetum ovati

24

38

Blechno spicantis-Quercetum roboris subas. pulmonarietosum longifoliae

15

Bromelio chrysanthae-Platymiscietum pinnati $\mathbf{3 1}$

Bromelio chrysanthae-Platymiscietum pinnati subas. duguetietosum riberensis

31

Bromo cathartici-Coronopodetum didymi $\quad 31$

Bryo pseudotriquetri - Plagiomnietum undulatae 7
Bupleuro gibraltarici-Pistacietum lentisci subas. adenocarpetosum telonensis

18

Bupleuro gibraltarici-Pistacietum lentisci subas. buxetosum balearicae

13

Bupleuro gibraltarici-Pistacietum lentisci subas. viburnetosum tini

29

Bursero graveolentis-Prosopidion pallidae $\quad 27$

Caesalpinio spinosae-Myrcianthetum ferreyrae 34

Calamintho baeticae-Gallietum scabri

18

Calamintho baeticae-Gallietum scabri subas. digitaletosum bocquetii

18

Calamintho baeticae-Gallietum scabri subas. lamietosum flexuosi

18

Calicotometum villosae subas. Juniperetosum turbinatae

21

Calicotomo intermediae-Maytenetum senegalensis

26

Calicotomo intermediae-Maytenetum senegalensis subas. sideritidetosum pusillae

26

Calicotomo villosae-Genistetum hirsutae subas. genistetosum triacanthi

18

Callitrichetum regis-jubae

24

Callitricho stagnalis-Ranunculetum saniculifolii24

Campanulion velutinae

15

Cardamino resedifoliae-Primuletum hirsutae $\mathbf{2 4}$

Cardamino resedifoliae-Primuletum hirsutae subas. potentilletosum alchimilloidis

24

Cardamino resedifoliae-Primuletum hirsutae subas. saxifragetosum iratianae

24

Carduo carpetani-Onopordetum acanthii $\mathbf{1 1}$

Carduo carpetani-Onopordetum acanthii subas. carduetosum carpetani $\quad \mathbf{1 1}$

Caricetum candicantis $\quad 29$

Caricion tartessianae $\quad 33$

Carici-Quercetum canariensis subas. holcetosum

Carico candicantis-Caesalpinietea spinosae $\mathbf{3 4}$

Centaureenion clementei $\mathbf{3 4}$

Centaureo baeticae-Carlinetum corymbosae $\mathbf{2 3}$

Centaureo sempervirentis-Quercetum suberis $\mathbf{3 3}$

Cercidio praecocis-Prosopidetum pallidae $\quad 27$

Cestro aurieulati-Prunetalia rigidae $\quad 27$

Chaenorrhino reyesi-Campanuletum fastigiatae subas. avellinietosum festucoidis $\quad 38$

Chaenorrhino villosae-Campanuletum mollis subas. athamantetosum vayredanae $\mathbf{2 3}$

Chaetantherion sphaeroidalis $\quad \mathbf{2 8}$

Cheilantho maderensis-Cosentinietum velleae 14

Cheilantho maderensis-Cosentinietum velleae subas. cheilanthetosum acrosticae 14

Chersodomo diclinae-Valerianetum nivalis $\mathbf{2 8}$

Chusqueetalia tessellatae $\quad 27$

Cirsio pyrenaici-Senecietum laderoi 29

Cirsio pyrenaici-Senecietum laderoi subas. primuletosum veris

29

Cistancho phelipeae -Suaedetum verae subas. limoniastretosum 
Cistancho phelipeae -Suaedetum verae subas. typicum

Cistetum africani-monspeliensis subas.

Cistetum africani-monspeliensis subas. genistetosum lanuginosae

24

Cistetum africani-monspeliensis subas. genistetosum triacanthi

24

Cistetum africani-monspeliensis subas. genistetosum umbellatae

24

Cistetum africani-monspeliensis subas. halimietosum serpentinicolae

24

Cisto clusii-Ulicetum (parviflorii) rivasgodayanii

14

Cisto clusii-Ulicetum (parviflorii) rivasgodayanii subas. anthyllidetosum plumosae

14

Cisto clusii-Ulicetum (parviflorii) rivasgodayanii subas. odontitosum purpureae

14

Cisto clusii-Ulicetum rivasgodayanii suabs. genistetosum retamoides

Cisto clusii-Ulicetum rivasgodayanii subas. lavanduletosum lanatae

Cisto salvifolii-Ulicetum australis

18

Cisto salvifolii-Ulicetum australis subas. lavanduletosum sampaianae

Citharexylo flexuosi-Crotonetalia alnifolii $\quad \mathbf{3 4}$

Cneoro triccoci-Buxetum balearicae subas. ceratonietosum siliquae

29

Coccolobietalia obtusifoliae

Coccolobietea obtusifoliae

Coccolobion obtusifoliae

Cololejeuneo-Rhynchostegietum tenellae

Commelinetalia fasciculatae

Cortaderietum jubatae

Crataego brevispinae-Populetum albae

Cresso truxillensis-Distichlietum spicatae

Crithmo-Limonietum auriculae-ursifolii

Cypero laevigati-Distichlietum spicatae

Cytiso baetici-Arbutetum unedi subas. bupleuretosum fruticosi

Cytiso baetici-Arbutetum unedonis

15

Cytiso baetici-Arbutetum unedonis subas. quercetosum cocciferae

15

Cytiso fontanesii-Genistetum ramosissimae $\mathbf{2 9}$

Cytiso plumosi-Ulicetum baetici subas. erinaceetosum

Cytiso plumosi-Ulicetum baetici subas. genistetosum equisetiformis

Cytiso plumosi-Ulicetum baetici subas. ulicetosum baetici

23

Cytiso tribracteolati-Juniperetum oxycedri $\quad \mathbf{2 4}$

Daphno hispanicae-Festucetum capillifoliae subas. stipetosum tenacissima

13

Daphno latifoliae-Aceretum granatensis subas. pistacietosum terebinthi

15

Deschampsio strictae-Agrostietum curtisii

Diantho merinoi-Plantaginetum radicatae $\mathbf{1 0}$

Diplostephio tacorensis-Parastrephietum lepidophyllae

28

Diplostephio tacorensis-Parastrephietum lepidophyllae subas. corryocactetosum brevistyli

28

Diplostephio tacorensis-Parastrephietum lepidophyllae subas. polylepidetosum besseri $\mathbf{2 8}$

Diplostephio tovari-Festucetum orthophyllae $\mathbf{3 4}$

Dorycnio recti-Salicetum pedicellatae 29

Dorycnio recti-Salicetum pedicellatae subas. nerietosum oleandri

29

Dunalio spitiosae-Baccharidetum latifoliae $\mathbf{2 8}$

Dunalio spitiosae-Baccharidetum latifoliae subas. calanzagrostietosum intermediae $\quad \mathbf{2 8}$

Dunalio spitiosae-Baccharidetum latifoliae subas. otholobietosum pubescentis $\quad \mathbf{2 8}$

Echinodorion boliviani $\quad 33$

Echinopsio chalaensis-Randietum armatae $\quad \mathbf{3 4}$

Echio boissieri-Antirrhinetum majii $\quad 31$

Eleocharitetalia minimae $\quad \mathbf{3 1}$

Eleocharitetum pachycarpae $\quad \mathbf{2 3}$

Elymo repentis-Phalaridetum coerulescentis $\mathbf{2 3}$

Equiseto gigantei-Salicetum humboldtianae $\mathbf{2 9}$

Equiseto telmateiae-Salicetum pedicellatae subas. salicetosum albae

21

Equiseto telmateiae-Salicetum pedicellatae subas. salicetosum angustifolia

24

Erico australis-Cistetum populifolii subas. halimietosum viscosii

Erico mediterraneae-Salicetum pedicellatae subas. galietosum viridiflori 29

Erico terminalis-Salicetunt eleagni $\mathbf{2 6}$

Erodio daucoidis-Saxifragetum erioblastae subas. erodietosum cheilanthifolii $\quad 29$

Erodio pulverulenti-Vulpietum membranaceae $\mathbf{3 5}$

Eryngio corniculati-Isoetetum setacei $\quad 34$

Eryngio dilatati-Jasonietum tuberosae $\mathbf{2 5}$

Espostoo melanostelis-Neoraimondion arequipensis

27

Feruletum communis $\quad 33$

Ferulo tingitanae-Carthametum arborescentis $\mathbf{2 5}$

Ferulo tingitanae-Carthametum arborescentis subas. smyrnietosum olusatri $\mathbf{2 5}$

Festuco multispiculatae-Agrostietum curtisii $\mathbf{3 3}$

Festuco multispiculatae-Arrhenatheretum sardoi

Festuco scariosae-Helictotrichetum arundani subas. festucetosum capillifoliae

Ficario-Fraxinetum angustifoliae subas. rosetosum corymbifera

Ficario-Fraxinetum angustifoliae subas. tamaricetosum africanae

Ficario-Fraxinetum angustifoliae subas. ulmetosum minoris

Fissidentetum taxifolii subas. rhizomnietosum punctati

Frangulo baeticae-Rhododendretum pontici subas. culcitetosum macrocarpae 
Fraxino angustifoliae-Quercetum pyrenaicae $\mathbf{1 3}$

Fraxino angustifoliae-Quercetum pyrenaicae subas. loniceretosum xylostei

13

Fraxino angustifoliae-Quercetum pyrenaicae subas. serratuletosum tinctoriae

Fumano juniperinae-Cistetum crispi

Galio baetici-Thymetum granatensis subas. convolvuletosum boissieri

Galio pruinosi-Dianthetum lusitani

29

Gaudinio fragilis-Agrostietum castellanae subas. agrostietosum pourretii

33

Gaudinio fragilis-Agrostietum castellanae subas. hypochaeridetosum platylepidis

22

Gaudinio fragilis-Hordeion bulbosi

Genistenion viciosoi

22

Genistetalia haenseleri-ramosissimae

29

Genistetum haenseleri

29

Genistetum ramosissimo-demnatensis

Genistion haenselero-polyanthi

27

29

27

Genisto cinerascentis-Adenocarpetum argyrophylli

33

Genisto cinerascentis-Adenocarpetum argyrophylli subas. echinospartetosum iberici

33

Genisto hirsutae-Cistetum ladaniferi subas. cistetosum libanotis

22

Genisto hirsutae-Cistetum ladaniferi subas. halimietosum viscosii

18

Genisto hystricis-Echinospartetum lusitanici subas. ericetosum cinereae

10

Genisto sanabrensis-Juniperetum nanae subas. echinospartetosum iberici

15

Genisto sanabrensis-Juniperetum nanae subas. vaccinietosum uliginosi

15

Genisto tridentis-Stauracanthetum boivinii $\mathbf{1 8}$

Genisto tridentis-Stauracanthetum boivinii suabs. cytisetosum tribracteolati

24

Genisto tridentis-Stauracanthetum boivinii subas. quercetosum lusitanicae

18

Genisto tridentis-Stauracanthetum boivinii subas. ulicetosum baetici

Genisto viciosoi-Velletum spinosae

23

Genisto viciosoi-Velletum spinosae subas. anthyllidetosum tejedensis

29

Geophiletum repentis

31

Geranio pusilli-Theligonetum cynocrambis subas. geranietosum purpurei

25

Glycerio declinatae-Catabrosetum aquaticae subas. alopecuretosum aequali

13

Gnaphalio-Polygonetum hydropiperoidis $\quad \mathbf{2 3}$

Grindelio bolivianae-Corryocactetum puquiensis

Grindelion glutinosae

34

Haageocereo limensis-Neoraimondietum arequipensis

Haageocereo limensis-Neoraimondietum arequipensis

29

Haageocereo limensis-Neoraimondietum arequipensis subas. plucheetosum chingoyonis

Haageocereo limensis-Neoraimondietum arequipensis subas. puyetosum ferrugineae

29

Hedysaro coronarii-Phalaridetum coerulescentis

Hedysaro-Phalaridetum glossopappetosum macroti

24

Helianthemo glabrati-Hypertcetum ericoidis $\mathbf{2 4}$

Helictero guazumifoliae-Bauhinietum benthamianae

31

Helodo-Sphagnetum subas. sphagnetosum crassicladi

Hieracio texedensis-Jasionetum minutae subas. centranthetosum nevadensis

29

Hoffmannseggio mirandae-Palauetum weberbaueri

34

Holco setiglumis-Anthoxanthetum aristati subas. sisymbrietosum officinalis

33

Hydrocleydo nymphoidis-Nymphoidetum herzogii

Hyperico caprifolii-Schoenetum nigricantis subas. ericetosum terminalis

26

Hyperico perforati-Ferulion communis 33

Imperato cylindricae-Scirpetum holoschoeni 27

Inulo crithmoidis - Arthrocnemetum glauci subas. typicum

3

Inulo crithmoidis - Arthrocnemetum glauci subas. suaedetosum verae

Ipomoeo fistulosae-Sennetum aculeatae $\quad 33$

Jasiono penicillatae-Linarietum saturejoidis subas. linarietosum (salzmannii) flavae

Jasonio saxatilis-Antirrhinetum mollis $\quad \mathbf{2 8}$

Junco rugosi-Ericetum andevalensis $\quad \mathbf{1 4}$

Jungietum axillaris $\quad 29$

Junipereta macrocarpae $\quad \mathbf{1 5}$

Juniperetum sabino-thuriferae subas. quercetosum fagineae $\mathbf{1 5}$

Koelerio dasyphyllae-Arenarietum erinacei $\mathbf{2 7}$

Lavandulo caesiae-Genistetum equisetiformi subas. calicotometosum intermediae

22

Lavandulo lanate-Ulicetum baetici subas. salvietosum lavandulifoliae $\quad 23$

Lavandulo multifidae-Stipetum tenacissimae $\mathbf{3 3}$

Lavandulo multifidae-Stipetum tenacissimae subas. helianthemetosum almeriensis

33

Lavandulo sampaianae-Cistetum populifoli $\mathbf{1 4}$

Lavandulo sampaianae-Genistetum hystricis subas. ericetosum aragonensis

14

Lavandulo sampaianae-Genistetum hystricis subas. halimietosum ocymoidis

14

Lavandulo sampaionae-Stauracanthetum lusitanicae

36

Limonietum emarginati subas. helichrysetosum rupestri

25

Limonietum emarginati subas. limonietosum 
sinuati

Limonio cossoniani-Sarcocornietum fruticosae $\mathbf{2 7}$

Limonio-Lycietum intricati subas. limonietosum gummiferae

Linario anticariae-Centaureetum clementei

Linarion pedunculatae

Lippio nodiflorae-Paspaletum vaginati

Lippio nodiflorae-Paspaletum vaginati subas. schoenoplectetosum olneyi

Loasion urentis

Loto-Cyperetum eragrostidae

Ludwigio octovalvis-Paspalion vaginati

Lythro juncei-Cyperetum fuscae

Malcolmio-Vulpietum alopecuri

Malcomio trilobae-Hymenocarpetum hamosi subas. loeflingietosum baeticae

Matucano haynei-Tillandsietum humilis

Minuartio hybridae-Saxifragetum tridactylitis 9

Mutisio acuminatae-Baccharidetalia lanceolatae

Mutisio acuminatae-Ophryosporion peruviani 27

Myrcianthion quinquelobae

Myrico pubescentis-Alnion acuminatae $\quad 27$

Myriophylletum quitensis subas. elodeetosum potamogetonis

28

Myrto communis-Quercetum suberis subas. halimietosum halimifolii

18

Myrto communis-Quercetum suberis subas. lavanduletosum luisieri

18

Myrto-Quercetum suberis subas. pteridetosum $\mathbf{1 5}$

Neckeradelphetum menziesii

Nectandro-Viticion

3

Neoporterio islayensis-Neoraimondietum arequipensis

Nicotianetalia paniculato-glutinosae

27

Nolano spathulatae-Palauetum dissectae $\quad 34$

Notholaeno marantae-Cheilanthetum guanchicae

14

Notholaeno marantae-Cheilanthetum guanchicae subas. cheilanthetosum maderensis

14

Nototricho obcuneatae-Xenophylletum poposi $\mathbf{2 8}$

Nototricho obcuneatae-Xenophylletum poposi subas. valerianetosum nivalis

28

Nototrichon obcuneatae

28

Odontito purpureae-Thymetum baetici subas. lavanduletosum lanatae

29

Oleo sylvestris-Quercetum broteroi

24

Ononido variegatae-Linarietum pedunculatae 13

Ononido variegatae-Linarietum pedunculatae subas. linarietosum pygmeae

Ornithopo isthmocarpi-Anthoxantetum ovati $\quad 27$

Ornithopo isthmocarpi-Anthoxantetum ovati subas. vulpietosum alopecurori

27

Oryzion perennis

31

Oryzo grandigiumis-Hymenachnetalia amplexicaulis

Oryzopsi-Antirrhinetum granitici subas. chelidonietosum majoris

16

Oryzopsi-Antirrhinetum granitici subas. lobularietosum maritime $\mathbf{1 6}$

Oryzopsi-Antirrhinetum granitici subas. vaillantietosum murale

Oryzopsio-Daucetum maximi subas. glaucietosum flavi

Oxycaryo cubensis-Eleocharitetum acutangulae

Paeonio broteroi-Abietetum pinsaponis subas. juniperetosum phoeniceae

Paeonio coriaceae-Quercetum rotundifoliae subas. arbutetosum unidonis

15

Parapholido incurvae-Frankenietum laevis $\mathbf{2 5}$

Parastrephio lucidae-Festucelum orthophyllae $\mathbf{2 8}$

Parastrephio lucidae-Festucelum orthophyllae subas. azorelletosum compactae $\mathbf{2 8}$

Parastrephio lucidae-Festucelum orthophyllae subas. polylepidetosum tarapacanae $\quad \mathbf{2 8}$

Parietarietum judaicae subas. cymbalarietosum muralis

Parietarietum judaicae subas. phagnaletosum saxatilis

Pellio epiphyllae - Plagiomnietum rostratae 7

Peperomio galioidis-Puyion ferrugineae $\quad 27$

Peperomio galioidis-Tillandsietum usneioidis 27

Phalacrocarpo oppositifoliae-Saxifragetum continentalis

Phalaridetalia coerulescentis

Philoglosso peruvianae-Urocarpidelum peruviani

Phleo nodosi-Poetum bulbosae subas. trifolietosum repentis

Phyllireo angustifoliae-Quercetum lusitanicae subas. quercetosum rotundifoliae $\mathbf{1 8}$

Pinguiculo grandiflorae-Caricetum lepidocarpae subas. caricetosum rostratae

13

Pino halepensis-Juniperetum phoeniceae $\quad 23$

Pino halepensis-Juniperetum phoeniceae suabs. genistetosum haenseleri $\quad \mathbf{2 3}$

Pino pinastri-Juniperion phoenicea $\quad 23$

Pino pinastri-Quercetum cocciferae 14

Pityrogrammo trifoliatae-Baccharidion salicifoliae

29

Plantagini albicantis-Stipetum parviflorae $\quad \mathbf{2 1}$

Plantago loeflingii-Prolongoetum pectinata 9

Plucheetum absinthioidis $\quad 34$

Plucheion absinthioidis $\quad 34$

Polyachyro sphaerocephali-Puyetum densiflorae34

Polylepidetalia racemosae $\quad 27$

Polypodio pycnocarpi-Puyetalia ferrugineae 27

Poo annuae-Spergularietum salinae 13

Poo sylvicolae-Festucetum atlantigenae $\quad 22$

Poo sylvicolae-Festucetum atlantigenae subas. scirpetosum maritimi $\quad 22$

Potentillo maurae-Campanulion filicaulis $\mathbf{2 2}$

Poterio agrimonioidis-Quercetum suberis subas. 
quercetosum pyrenaicae

33

Pruno mahalebo-Berberidetum hispanicae subas. prunetosum postratae

23

Puccinellio maritimae - Arthrocnemetum perennis subas. arthrocnetnetosum fruticosi

Pulicario uliginosae-Agrostietum salmanticae subas. cynodontetosum dactylionis

33

Pyre bourgaeanae-Quercetum rotundifoliae subas. myrtetosum communis

15

Pyre bourgaeanae-Quercetum rotundifoliae subas. quercetosum fugineae

15

Pyro bourgeanae-Quercetum rotundifoliae subas. jasminetosum fruticantis

33

Pyro bourgeanae-Quercetum rotundifoliae subas. juniperetosum badiae

33

Pyro bourgeanae-Quercetum rotundifoliae subas. quercetosum pyrenaicae

33

Querco-Teucrietum siculi subas. polystichetosum settferi

15

Querco-Teucrietum siculi subas. teuerietosum siculi

Randio venezuelensis-Annonetum jahnii

31

Ranunculetum limoselloidis

28

Ranunculetum tripartiti

24

Ranunculion bullati

33

Ranunculo hederacei-Callitrichetum stagnalis 27

Ranunculo macrophylli-Juncetum inflexi $\quad 23$

Ranunculo peltati-Callitrichetum brutiae $\quad 27$

Raphano raphanistri-Diplotaxietum catholicae $\mathbf{3 3}$

Resedo albae-Chrysanthemetum coronarii subas. cladanthetosum arabici

38

Rhamno myrtifolii-Juniperetum phoeniceae suabs. abietetosum pinsaponis

23

Rhamno pumili-Saxifragetum granatensis

23

Rhamno pumili-Saxifragetum granatensis subas. galietosum pulvinati

23

Rhododendretalia pontici

26

Rhododendro pontici-Prunetalia lusitanicae $\mathbf{2 4}$

Rhododendro pontici-Prunetalia lusitanicae $\mathbf{2 5}$

Rhododendro pontici-Prunetalia lusitanicae subas. culcitetosum macrocarpae

25

Rhododendro pontici-Prunetalia lusitanicae subas. daphnetosum latifoliae

25

Rhododendro pontici-Prunion lusitanicae

Rhododendro pontici-Prunion lusitanicae

24

25

Ribesido brachybotrys-Polylepidion besseri $\quad 27$

Rubetum idaeo-radulae

33

Rubetum ulmifolio-caesii

33

Rubetum ulmifolio-vigoi

33

Rubio tinctorum-Populetum albae subas. salicetosum pedicellatae

Rubo vigoi-Sambucetum nigrae

Rumici angiocarpi-Coleostephetum myconis $\mathbf{3 3}$

Rumici angiocarpi-Coleostephetum myconis subas. brassicetosum barrelieri

33

Rupicapnetum africanae

Rupicapnetum decipientis
Rusco-Quercetum canariensis subas. Quercetosum broteroi

Salicetalia caroliniano-humboldtianae $\quad 27$

Salicion pedicellatae $\quad 24$

Sanfragetum camposii $\quad \mathbf{1 5}$

Sarcocapnetum baeticae $\quad 23$

Sarcocornietalia neei $\quad 34$

Sarcocornio neei-Distichlion spicatae 34

Saturejo malacitanae-Coridothymetum capitati 26

Saturejo malacitanae-Coridothymetum capitati subas. sideritidetosum pusillae $\quad 26$

Saxifragetalia magellanicae $\quad 27$

Saxifragion magellanicae $\quad 27$

Schino mollis-Acacielum macracanthae $\quad 27$

Schino mollis-Acacietum macracanthae subas. baccharidetosum salicifoliae

29

Schino mollis-Tecometum arequipensis $\quad 34$

Schino mollis-Tecometum tanaceiiflorae $\quad 34$

Schoenoplectetalia olneyi-americani 34

Schoenoplectetum olneyi-americani 34

Scillo autumnalis-Ranunculetum bullati 33

Scrophulario glabratae-Rubion ulmifolii $\quad 37$

Scrophulario laxiflorae-Rhododendretum pontici

25

Scrophulario laxiflorae-Rhododendretum pontici

Sedetum granatensis-acris

Sedion caespitosi $\quad \mathbf{3 0}$

Sedo anglici-Thymetum caespititii 21

Sedo caespitosii-Crassuletum tillaeae subas. poetosum bulbosae $\quad 33$

Sedo wilczekiani-Sonchetum masguindalii $\quad 22$

Seselido vayredanae-Sarcocapnetum enneaphyllae

Seselietum vayredani subas. teucritosum rotundifoliae

15

Seselietum vayredani subas. teucritosum rotundifoliae

Seselio granatensis-Festucetum hystricis subas. arenarietosum erinacei

23

Sicyo baderoae-Urticion magellanicae $\quad 27$

Sideritido juryi-Hypericetum ericoidis $\quad 24$

Siegesbeckio serratae-Cotnmelinion fasciculatae

Sileno acutifoliae-Dianthetum lusitani 27

Sileno ramosissimae-Cutandietum maritimae

Sisymbrio irio-Malvetum parviflorae

Smilaco mauritanicae-Quercetum rotundifoliae subas. quercetosum suberis $\quad \mathbf{1 5}$

Solpichroetalia glandulosae $\mathbf{2 8}$

Spartio juncei-Rubetum ulmifolii 33

Spergulario mediae-Puccinellietum fasciculatae 29

Sphaerothallietum fruticuloso-foliaceae

Sphaerothallio-Xanthoparmelion vaganti 4

Sporobolo virginici-Distichlietum spicatae $\mathbf{3 4}$

Sporobolo-Centaureetum sphaerocephalae 1

Stemmacantho ericeticolae-Schaenetum 
nigricantis

Stipion parviflorae

Stuckenietum punensis

Symploco nanae-Oreopanaxion rusbyi

Tamo-Oleetum sylvestris subas quercetosum suberis

Tecomion arequipensis

Teucrio baetici-Quercetum suberis subas. quercetosum rotundifoliae

Teucrio compacti-Cistetum ladaniferi subas. cistetosum populifolii

Teucrio edetani-Anthyllidetum terniflorae

Teucrio homotrichi-Ulicetum dianii

24

Teucrio homotrichierinacetosum anthyllidis

Teucrio homotrichi-Ulicetum dianii subas. ononidetosum fruticosae

Teucrio-Kerneretum boissieri subas. anthyllidetum ramburii

Teucrio-Linarietum lilacinae

Thymo gracile-Lavanduletum lanatae subas. genistetosum scorpii

29

Thymo gracile-Stipetum tenacissimae subas. stipetosum giganteae

14

Thymo longiflori-Salvietum vellereae subas. velletosum spinosae

29

Tortello flavovirentis - Bryetum dunensi

Trichostomo littoralis-Orthothecietum durieu $\quad 7$

Trifolio cherleri-Plantaginetum bellardii subas. anthoxanthetosum ovati

Tristerido peruviani-Myrcianthetum quinquelobae

Ulici baetici-Cistetum clusii subas. halimietosum halimifolii

Ulici borgiae-Calicotomenion villosae

22

21

7

$m$

8

.

.

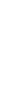

(3)

(1)

(5)

Ulici eriocladi-Cistetum ladaniferi subas. ericetosum 27

Ulici eriocladi-Cistetum ladaniferi subas. ericetosum australis

18

Ulici eriocladi-Ericetum andevalensis $\quad \mathbf{2 4}$

Vaccinio cylindracei-Rubetum hochstetterori $\mathbf{3 7}$

Vaccinio myrtilli-Juniperetum nanae subas. jasionetosum brevisepalae

15

Valeraniello-Bupleuretum lancifolii subas. arenarietosum cerastioidis

Valeriano thalictrioidis-Saxifragetum magellanicae

Valleo stipularis-Alnetum acuminatae $\quad 27$

Verbasco martinezii-Ononidetum ramosissimae subas. feruletosum tingitanae $\quad \mathbf{2 5}$

Vinco difformis-Quercetum fagineae $\quad \mathbf{3 4}$

Vinco difformis-Ulmetum minoris $\quad 37$

Violo (demetriae)—Ionopsidietum prolongoi $\mathbf{4}$

Violo henriquesli-Silenetum littoreae $\mathbf{1 3}$

Weberbauerocereo rahuii-Browningietum candelaris $\quad 34$

Weberbauerocereo rahuii-Corryocactetum brevistyli

Weberbauerocereo rahuii-Corryocactetum brevistyli subas. Crotonetosum ruiziani

Weberbauerocereo torataensis-Corryocactetum brevistyli

Weberbauerocereo weberbaueri-Corryocactetum brevistyli

Wernerio pygmaeae-Puccinellietum oresigenae $\mathbf{2 8}$

Wissadulo periplocifoliae-Cassion torae $\mathbf{3 1}$

Withanio frutescentis-Lycietum intricati subas. atriplicetosum glaucae

Withanio frutescentis-Lycietum intricati subas. lobularietosum columbretensis 